\title{
A posteriori error estimation techniques in practical finite element analysis
}

\author{
Thomas Grätsch, Klaus-Jürgen Bathe * \\ Department of Mechanical Engineering, Massachusetts Institute of Technology, 77 Massachusetts Avenue, Room 3-356, \\ Cambridge, MA 02139, USA
}

Received 29 December 2003; accepted 26 August 2004

\begin{abstract}
In this paper we review the basic concepts to obtain a posteriori error estimates for the finite element solution of an elliptic linear model problem. We give the basic ideas to establish global error estimates for the energy norm as well as goal-oriented error estimates. While we show how these error estimation techniques are employed for our simple model problem, the emphasis of the paper is on assessing whether these procedures are ready for use in practical linear finite element analysis. We conclude that the actually practical error estimation techniques do not provide mathematically proven bounds on the error and need to be used with care. The more accurate estimation procedures also do not provide proven bounds that, in general, can be computed efficiently. We also briefly comment upon the state of error estimations in nonlinear and transient analyses and when mixed methods are used.
\end{abstract}

(c) 2004 Elsevier Ltd. All rights reserved.

Keywords: Finite element analysis; A posteriori error estimation; Goal-oriented error estimation; Dual problem; Practical procedures

\section{Contents}

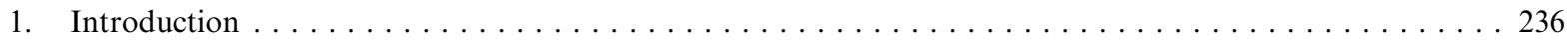

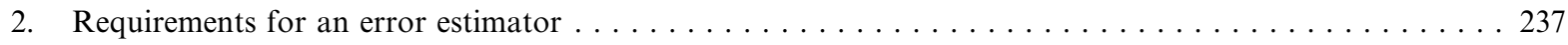

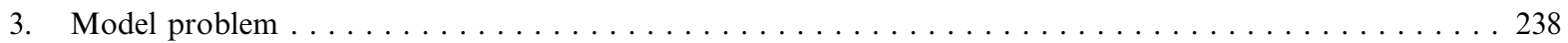

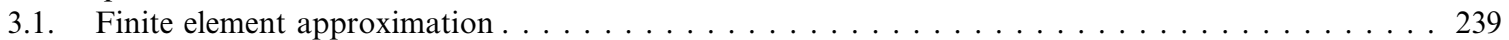

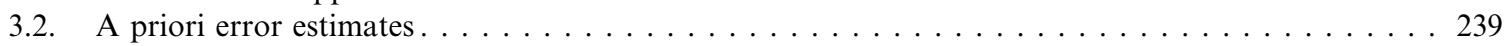

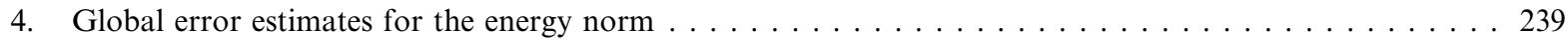

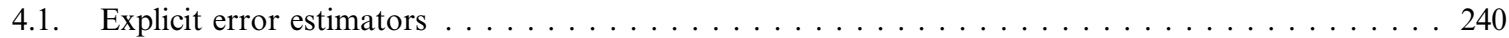

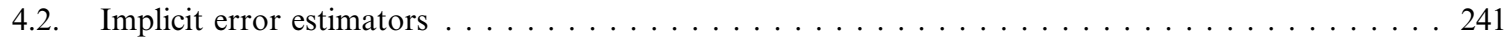

\footnotetext{
* Corresponding author. Tel.: +1 617253 6645; fax: +1 6172532275 .

E-mail address: kjb@mit.edu (K.J. Bathe).
} 


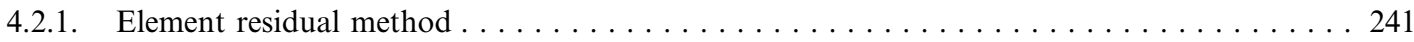

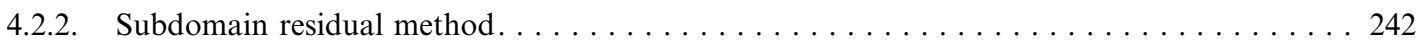

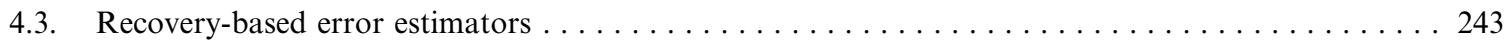

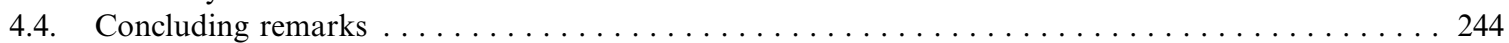

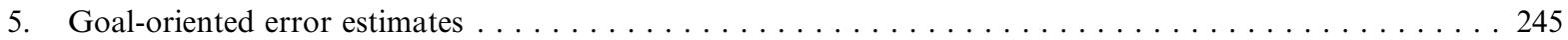

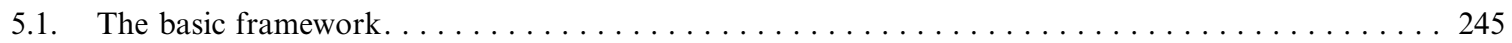

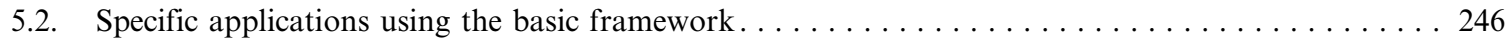

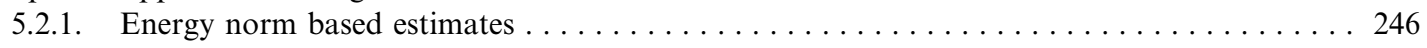

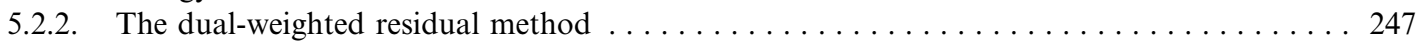

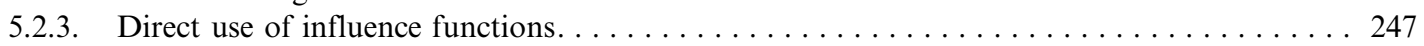

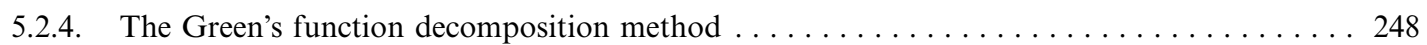

5.2.5. Exact-bounds approach . . . . . . . . . . . . . . . . . . . . . . . . . 249

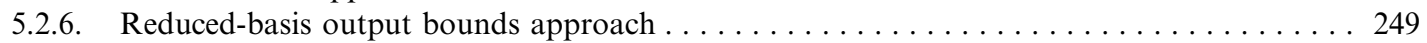

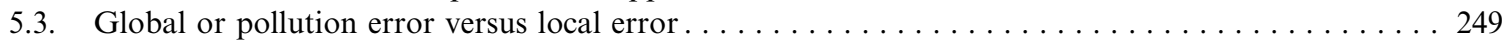

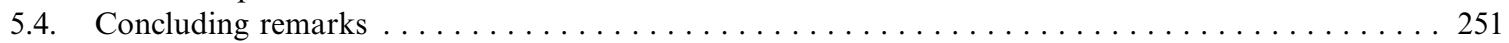

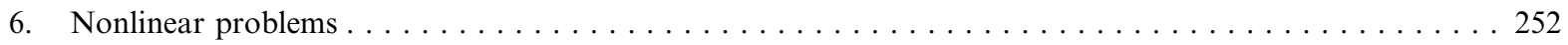

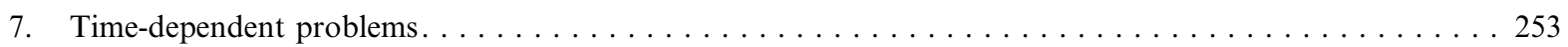

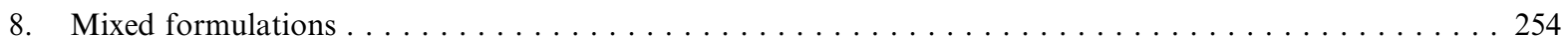

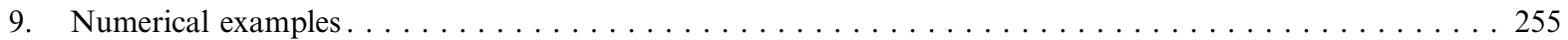

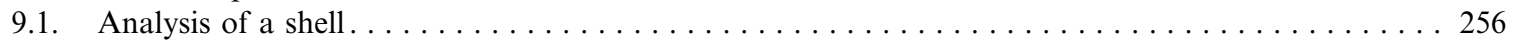

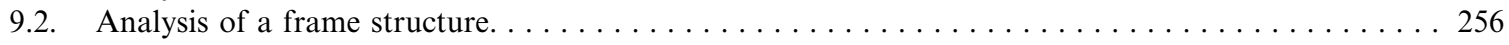

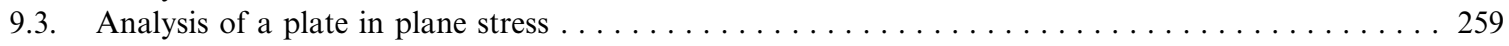

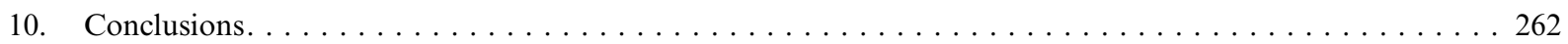

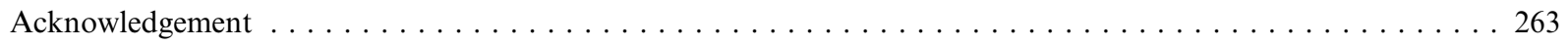

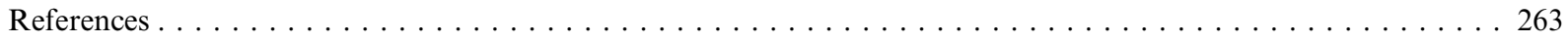

\section{Introduction}

The modeling of physical phenomena arising in engineering and the sciences leads to partial differential equations in space and time, expressing the mathematical model of the problem to be solved. In general, analytical solutions of these equations do not exist, hence numerical methods such as the finite element method are employed. A major feature of numerical methods is that they involve different sources of numerical errors $[1,2]$. The focus of this paper is only on the discretization error which is due to the finite element (polynomial) approximation of the solution. Hence, we assume that an appropriate mathematical model has been chosen and, even for this case, we are only concerned with one specific error, namely the discretization error arising in the finite element solution of this model.

Since the late 1970s several strategies have been developed to estimate the discretization errors of finite element solutions. Basically, there are two types of error estimation procedures available. So called a priori error estimators provide information on the asymptotic behavior of the discretization errors but are not designed to give an actual error estimate for a given mesh. In contrast, a posteriori error estimators employ the finite element solution itself to derive estimates of the actual solution errors. They are also used to steer adaptive schemes where either the mesh is locally refined ( $h$-version) or the polynomial degree is raised ( $p$-method). Most a posteriori error estimators developed prior to the mid-1990s focused on the global error in the energy norm. Then recently the theory was extended to estimate the error in particular quantities of interest. To understand the importance of this extension it must be realized that many local or global quantities of interest - such as deformations, stresses, drag and lift coefficients or the heat transfer of a structural part — can be obtained by applying a linear functional to the solution. 
This new development is commonly referred to as goaloriented error estimation since the aim is to provide error estimates and error bounds for particular quantities of interest.

In the following we will use the words "error estimates" and "error bounds". An error estimate denotes a quantity that is an approximation to the actual unknown error, whereas upper and lower error bounds are quantities that are always larger resp. smaller than the actual unknown error. Hence, error bounds can be guaranteed but still be inaccurate, whereas an error estimate should be accurate although, in general, it over- or underestimates the true error.

Today, a posteriori error estimates are well developed for a large class of simple linear elliptic model problems. The crucial question is whether these procedures are also effective in practical solutions such as in the linear analysis of geometrically complex 2D, 3D and shell problems, and in the analysis of problems including nonlinear effects, time-dependent loads or multi-physics phenomena. From a practical point of view, there is much interest in reliable and efficient methods to estimate the error in complex analyses. In this context we shall consider reliability to mean that the error estimates can be expected to be accurate, and efficiency to mean that the computer time to obtain these estimates is small when compared to the total processing time used. In reviewing the state of a posteriori error estimators, we will conclude that efficient error estimates still need to be used with care because they are generally not based on guaranteed error bounds. In fact, nearly-guaranteed error bounds are still quite expensive to evaluate for complex problems and are frequently not yet available.

The outline of the paper is as follows: We start with a summary of some basic requirements for an error estimator and give a simple schematic example to indicate what an ideal error estimator would give in practical engineering analysis. Next, we consider an elliptic linear model problem for which explicit and implicit error estimators as well as recovery-based error estimators for the global energy norm are presented. We then introduce for the same model problem goal-oriented error estimators which can be used to estimate the error in the calculation of arbitrary quantities of interest. For both, the global energy and goal-oriented error estimators, we give some concluding remarks on the application of these methods in the solution of complex but linear problems. Furthermore, we briefly comment upon error estimation techniques in nonlinear and time-dependent analyses as well as in the use of mixed methods. Then we present some demonstrative numerical solutions. Finally, we conclude with some general suggestions on future developments and open issues.

The theory of error estimation is large and this paper only presents some experiences. Indeed, we only aim to provide the reader with the main ideas of a posteriori error estimation and a state-of-the-art assessment regarding the use of these procedures when applied to practical problems. For more details on finite element error estimation procedures, and additional views and approaches, see for example Verfürth [3], Estep et al. [4], Ainsworth and Oden [5], Babuška and Strouboulis [6], and the references therein.

\section{Requirements for an error estimator}

The main purpose of any a posteriori error estimator is to provide an estimate and ideally bounds for the solution error in a specified norm or in a functional of interest if the problem data and the finite element solution are available. Some characteristics of an effective error estimator include:

- The error estimate should be accurate in the sense that the predicted error is close to the actual (unknown) error.

- The error estimate should be asymptotically correct in the sense that with increasing mesh density the error estimate should tend to zero at the same rate as the actual error.

- Ideally, the error estimator should yield guaranteed and sharp upper and lower bounds of the actual error.

- The error estimator should be computationally simple, with the error estimate (and bounds) inexpensive to compute when measured on the total computations of the analysis.

- The error estimator should be robust with regard to a wide range of applications, including nonlinear analysis.

- An implementation of the error estimator should be possible to steer an adaptive refinement process with the error estimate used to optimize the mesh with respect to the goal of the computation.

Of course, an ideal error estimator that meets all these requirements is not yet available. But even for linear problems, it is in general not possible to provide inexpensively computable and guaranteed error bounds which would be of much practical interest. To illustrate the requirements, we consider the static analysis of a plate with a hole acted upon by a longitudinal tensile load (see Fig. 1) where one-fourth of the plate is shown. Ideally, the application of error estimation procedures should result with little computational cost in an accurate error estimate, based ideally on sharp and guaranteed maximum absolute error bounds, for the displacements and the stresses at every point of the structure (i.e. for every quantity of interest) which we call the "ideal-error-estimator-solution" in finite element analysis. With the ideal-error-estimator-solution available, the analyst could refine the mesh in those regions 


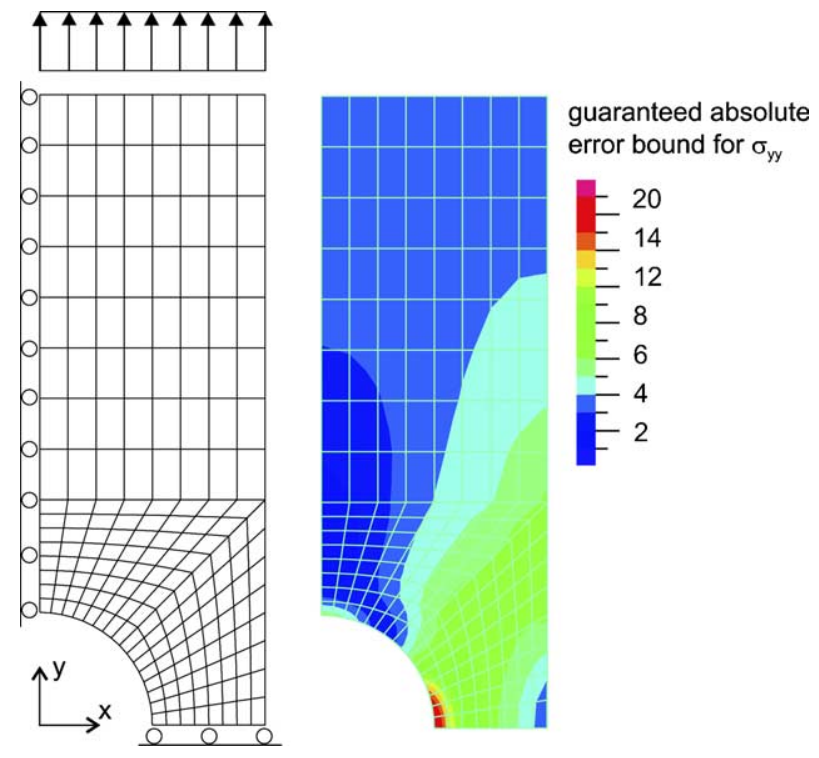

Fig. 1. The ideal-error-estimator-solution in finite element analysis: guaranteed maximum absolute error bounds at every point of the structure. Not available yet.

where the error is large in order to obtain an efficient mesh and be certain about the accuracy of the solution for any mesh used.

Of course, a key requirement for this error estimator to be useful in engineering practice is that the computational cost of the error estimate (and ideally the error bounds) must be much smaller than the added computational cost to simply use a very fine mesh.

Today, even when considering only linear analysis, we are far from the ideal-error-estimator-solution, since either error bounds for quantities of interest are guaranteed but expensive to compute or (mostly) not computable at all, or they are computable but not guaranteed. Also, the efficient computation of accurate and nearlyguaranteed error bounds achieved in the solution of simple model problems may actually be very expensive for complex engineering problems. Note that global error estimates for the energy norm consider only the error in the global energy norm and do not provide any local information.

In engineering practice, error measures are not yet much used and there are only a few contributions that address the application of effective error estimation procedures in the solution of complex problems of practical interest. We mention these contributions below.

\section{Model problem}

The elliptic linear model problem is Poisson's equation on a bounded, polyhedral and two-dimensional domain $\Omega \in \mathbb{R}^{2}$ with Lipschitz boundary $\Gamma=\Gamma_{\mathrm{D}} \cup \Gamma_{\mathrm{N}}$ where $\Gamma_{\mathrm{D}}$ and $\Gamma_{\mathrm{N}}$ are the Dirichlet and Neumann boundaries, respectively. The boundary value problem consists of finding the solution $u$ that satisfies

$$
\begin{gathered}
-\Delta u=f \quad \text { on } \Omega ; \quad u=0 \quad \text { on } \Gamma_{\mathrm{D}} ; \\
\mathbf{n} \cdot \nabla u=g \quad \text { on } \Gamma_{\mathrm{N}}
\end{gathered}
$$

The data are assumed to be sufficiently smooth, that is, $f \in L^{2}(\Omega), g \in L^{2}\left(\Gamma_{\mathrm{N}}\right)$ and $\mathbf{n}$ is the unit outward normal vector to $\Gamma$. An equivalent formulation of the boundary value problem is the variational formulation seeking $u \in V$ such that

$a(u, v)=l(v) \quad \forall v \in V$

where the trial and test space $V$ is the usual Sobolev space of functions from $H^{1}(\Omega)$ whose trace vanishes on the Dirichlet part of the boundary

$V=\left\{v \in H^{1}(\Omega): v=0\right.$ on $\left.\Gamma_{\mathrm{D}}\right\}$

The form $a(u, v)$ is assumed to be a $\mathrm{V}$-coercive bilinear form on $V(\Omega) \times V(\Omega)$ and the linear functional $l(v)$ is an element of the dual space $V^{\prime}(\Omega)$

$a(u, v)=\int_{\Omega} \nabla u \cdot \nabla v \mathrm{~d} \Omega$

$l(v)=\int_{\Omega} f v \mathrm{~d} \Omega+\int_{\Gamma_{\mathrm{N}}} g v \mathrm{~d} s$

Associated with the bilinear form is the energy norm defined by

$\|v\|_{E}=\sqrt{a(v, v)}$ 
As is well known, the existence and uniqueness of the variational solution is provided by the Lax-Milgram theorem which requires the bilinear form to be bounded and elliptic on $V(\Omega) \times V(\Omega)$

$|a(v, w)| \leqslant M\|v\|_{V}\|w\|_{V} \quad \forall v, w \in V \times V$

$a(v, v) \geqslant \alpha\|v\|_{V}^{2} \quad \forall v \in V$

where $M$ and $\alpha$ are positive constants independent of $v$ and $w$.

While we consider here a model problem with a scalar for solution, the concepts given in the paper are, of course, also applicable to the general linear elasticity problem by using the appropriate vectors of solution variables and the corresponding solution spaces. Indeed, in the example solutions in Section 9, we only consider more general elasticity problems.

\subsection{Finite element approximation}

The finite element formulation is based on the Bubnov-Galerkin procedure where $V_{h} \subset V$ is assumed to be a finite element subspace consisting of cellwise polynomial functions of order $p$ over the finite element partition $\mathscr{T}_{h}$. The partition, or simply mesh, formed by the union of all elements, is assumed to coincide exactly with the domain $\Omega$ and any two elements are either disjoint or share a common edge. Of course, the boundary of each element is also assumed to be Lipschitz-continuous. Then the finite element approximation means to find a function $u_{h} \in V_{h}$ such that

$a\left(u_{h}, v_{h}\right)=l\left(v_{h}\right)$

for all test functions $v_{h} \in V_{h} \subset V$. The error of the finite element approximation denoted by $e_{h}=u-u_{h}$ satisfies the error representation

$$
\begin{aligned}
a\left(e_{h}, v\right) & =a(u, v)-a\left(u_{h}, v\right) \\
& =l(v)-a\left(u_{h}, v\right)=\mathscr{R}_{h}(v) \quad \forall v \in V
\end{aligned}
$$

which is the basis for a large class of error estimators using the energy norm. Here, $\mathscr{R}_{h}(\cdot)$ is called the residual functional or the weak residual. If in (9) the choice of test functions is restricted to the finite element space, the fundamental Galerkin orthogonality condition follows $[1,7,8]$

$$
a\left(e_{h}, v_{h}\right)=\mathscr{R}_{h}\left(v_{h}\right)=0 \quad \forall v_{h} \in V_{h}
$$

Assuming that the bilinear form is positive definite it follows that

$$
\left\|\mathscr{R}_{h}\right\|_{V^{\prime}}=\sup _{v \in V(\Omega)} \frac{\left|\mathscr{R}_{h}(v)\right|}{\|v\|_{E}}=\left\|e_{h}\right\|_{E}
$$

where $\left\|\mathscr{R}_{h}\right\|_{V^{\prime}}$ denotes the norm of the residual in the dual space $V^{\prime}(\Omega)$.

\subsection{A priori error estimates}

A priori error estimates provide useful information on the asymptotic behavior of the approximation. The most important property of any conforming finite element formulation based on a symmetric bilinear form is the optimality condition

$\left\|u-u_{h}\right\|_{E}=\min \left\|u-v_{h}\right\|_{E} \quad \forall v_{h} \in V_{h}$

which states that there is no "better" approximation in the finite element space $V_{h}$ (i.e., that is closer to the exact solution) than the finite element solution itself, if the distance is measured in the energy norm [1]. For both symmetric and nonsymmetric variational problems there is another optimality condition called Céa's Lemma which asserts that for elliptic bilinear forms it holds

$\left\|u-u_{h}\right\|_{V} \leqslant \frac{M}{\alpha} \inf _{v_{h} \in V_{h}}\left\|u-v_{h}\right\|_{V}$

where $M$ and $\alpha$ are the constants defined in (6) and (7). Basically, Céa's Lemma asserts that the error of the finite element solution measured in the $V$-norm is of the same order as the interpolation error. Choosing $V \subset H^{1}$ and employing interpolation estimates it turns out that the error measured in the $H^{1}$-norm is of the order $O\left(h^{p}\right)$

$\left\|u-u_{h}\right\|_{H^{1}(\Omega)} \leqslant c h^{p}\|u\|_{H^{p+1}(\Omega)}$

where $c$ is a stability and interpolation constant which does not depend on the actual Ansatz (interpolation) space ${ }^{1}$ and $h$ denotes the maximum of all element sizes. Furthermore, we have for the error in the $L^{2}$-norm

$\left\|u-u_{h}\right\|_{L^{2}(\Omega)} \leqslant c h^{p+1}\|u\|_{H^{p+1}(\Omega)}$

which means that the convergence rate for the solution itself is $O\left(h^{p+1}\right)[1]$.

\section{Global error estimates for the energy norm}

In this section, we present various error estimators for the global error in the energy norm of the above mentioned elliptic model problem when a specific (not very fine) mesh has been used. Of course, it is always possible to solve problems (8) or (9) very accurately using a very fine mesh and then the (almost) exact error can be calculated. However, instead, we want to estimate the discretization error while not knowing the exact solution.

In order to evaluate the accuracy of any error estimate, we use the effectivity index defined by

\footnotetext{
1 Throughout this paper we use $c$ as a generic constant, which thus may take different values in successive occurrences, even in the same equation.
} 
$\lambda=\frac{E_{h}}{\left\|e_{h}\right\|_{E}}$

with $E_{h}$ denoting an estimate for the error in the energy norm. The effectivity index represents the degree of overor underestimation and should be ideally close to 1.0 .

Error estimators that are based directly on the finite element approximation and the data of the problem are usually referred to as explicit error estimators. In contrast, implicit error estimators require the solution of auxiliary local boundary value problems. Hence, explicit error estimators in general require less computational effort than implicit schemes, but they involve compromises in robustness and accuracy. A third class of error estimators are the recovery-based error estimators. The main idea of these error estimators is to smooth the gradients of the solution and compare the unsmoothed and the smoothed gradients in order to assess the solution error.

\subsection{Explicit error estimators}

Explicit error estimators involve a direct computation of the interior element residuals and the jumps at the element boundaries to find an estimate for the error in the energy norm, see the fundamental work of Babuška and Rheinboldt [9,11], Babuška and Miller [10] and Kelly et al. [12]. The starting point is the error representation

$a\left(e_{h}, v\right)=l(v)-a\left(u_{h}, v\right) \quad \forall v \in V$

which holds true for arbitrarily chosen test functions $v \in V$. If the domain integral is split into the contributions from each element, (17) can be rewritten for our model problem as

$$
\begin{aligned}
a\left(e_{h}, v\right)= & \sum_{K \in \mathscr{T}_{h}}\left\{\int_{K} f v \mathrm{~d} \Omega+\int_{\partial K \cap \Gamma_{\mathrm{N}}} g v \mathrm{~d} s\right. \\
& \left.-\int_{K} \nabla u_{h} \cdot \nabla v \mathrm{~d} \Omega\right\} \quad \forall v \in V
\end{aligned}
$$

where $K$ denotes the volume of an element in $\mathscr{T}_{h}$ and $\partial K$ denotes its boundary. Applying integration by parts to the last term in (18) and rearranging terms leads to

$a\left(e_{h}, v\right)=\sum_{K \in \mathscr{T}_{h}} \int_{K} R v \mathrm{~d} \Omega+\sum_{\gamma \in \partial_{\mathscr{T}}} \int_{\gamma} J v \mathrm{~d} s \quad \forall v \in V$

where $R$ is the interior element residual

$R=f+\Delta u_{h} \quad$ in $K$

and $J$ is the jump of the gradient across the element edge $\gamma$

$J= \begin{cases}\left(\mathbf{n} \cdot \nabla u_{h}+\mathbf{n}^{\prime} \cdot \nabla u_{h}^{\prime}\right) & \text { if } \gamma \not \Gamma \\ g-\mathbf{n} \cdot \nabla u_{h} & \text { if } \gamma \subset \Gamma_{\mathrm{N}} \\ 0 & \text { if } \gamma \subset \Gamma_{\mathrm{D}}\end{cases}$ where on interelement edges, $\gamma \nsubseteq \Gamma$, the edge $\gamma$ separates elements $K$ and $K^{\prime}$.

Next, we utilize the Galerkin orthogonality condition (10) to introduce the interpolant $\mathscr{I}_{h} v$ into (19) which results in

$$
\begin{aligned}
a\left(e_{h}, v\right)= & \sum_{K \in \mathscr{T}_{h}} \int_{K} R\left(v-\mathscr{I}_{h} v\right) \mathrm{d} \Omega \\
& +\sum_{\gamma \in \partial \mathscr{T}_{h}} \int_{\gamma} J\left(v-\mathscr{I}_{h} v\right) \mathrm{d} s \quad \forall v \in V
\end{aligned}
$$

Applying the Cauchy-Schwarz inequality elementwise yields

$$
\begin{aligned}
a\left(e_{h}, v\right) \leqslant & \sum_{K \in \mathscr{T}_{h}}\|R\|_{L^{2}(K)}\left\|v-\mathscr{I}_{h} v\right\|_{L^{2}(K)} \\
& +\sum_{\gamma \in \partial \mathscr{T}_{h}}\|J\|_{L^{2}(\gamma)}\left\|v-\mathscr{I}_{h} v\right\|_{L^{2}(\gamma)}
\end{aligned}
$$

According to results of interpolation theory we have

$\left\|v-\mathscr{I}_{h} v\right\|_{L^{2}(K)} \leqslant c h_{K}\|v\|_{H^{1}(\tilde{K})}$

$\left\|v-\mathscr{I}_{h} v\right\|_{L^{2}(\partial K)} \leqslant c \sqrt{h_{K}}\|v\|_{H^{1}(\tilde{K})}$

where $h_{K}$ is the diameter of the element $K$. The symbol $\tilde{K}$ denotes the subdomain of elements sharing a common edge with $K$ and $c$ is an unknown interpolation constant which depends for our model problem on the shape of the elements. Using these estimates in (23) leads to

$a\left(e_{h}, v\right) \leqslant c\|v\|_{H^{1}(\Omega)}\left\{\sum_{K \in \mathscr{T}_{h}} h_{K}^{2}\|R\|_{L^{2}(K)}^{2}+\sum_{\gamma \in \partial \mathscr{T}_{h}} h_{K}\|J\|_{L^{2}(\gamma)}^{2}\right\}^{1 / 2}$

Employing the inequality $\|v\|_{H^{1}(\Omega)} \leqslant c\|v\|_{E}$ and substituting $e_{h}$ in place of $v$ yields the final error bound

$\left\|e_{h}\right\|_{E}^{2} \leqslant c\left\{\sum_{K \in \mathscr{T}_{h}} h_{K}^{2}\|R\|_{L^{2}(K)}^{2}+\sum_{\gamma \in \partial \mathscr{T}_{h}} h_{K}\|J\|_{L^{2}(\gamma)}^{2}\right\}$

where apart of the constant $c$ all quantities on the righthand side of (27) can be calculated explicitly. In practice (27) is regrouped as

$\left\|e_{h}\right\|_{E}^{2} \leqslant \sum_{K \in \mathscr{T}_{h}}\left\{c_{1} h_{K}^{2}\|R\|_{L^{2}(K)}^{2}+c_{2} h_{K}\|J\|_{L^{2}(\partial K)}^{2}\right\}$

where the constant $c$ is split into contributions $c_{1}$ and $c_{2}$ corresponding to the element residual and the jump terms, respectively. We also note that on interelement boundaries $\partial K \nsubseteq \Gamma$ the jump $J$ is multiplied by the factor $1 / 2$ to distribute the error equally onto the two elements sharing the common edge.

The expression in (28) directly leads to a local error indicator $\eta_{K}$ defined by

$$
\begin{aligned}
& \left\|e_{h}\right\|_{E}^{2} \leqslant\left(E_{h}\right)^{2}=\sum_{K \in \mathscr{T}_{h}} \eta_{K}^{2} \\
& \text { with } \eta_{K}^{2}=c_{1} h_{K}^{2}\|R\|_{L^{2}(K)}^{2}+c_{2} h_{K}\|J\|_{L^{2}(\partial K)}^{2}
\end{aligned}
$$


Of course, the constants $c_{1}$ and $c_{2}$ in (29) are in general unknown. However, research has been conducted to evaluate these constants for specific problems - considering more general cases than our model problem - but the values obtained are related to worst-case scenarios and the error bound is generally not sharp. For instance, Johnson and Hansbo solve in [13] an additional eigenvalue problem in order to obtain computable values for the constants. In case of shell elements, the residual terms in (28) consist of bending, membrane and shear parts so that different weighting factors for each of these parts have to be applied (see e.g. Ref. [14]) and specific care is necessary regarding the "locking" phenomenon [1]. For general linear elastic analysis, the constants are unknown and cannot be calculated with a reasonable computational effort. However, we should point out that using (23) instead of (22) has significant consequences regarding the accuracy of any subsequent error estimate or bound, since the cancellation of errors over the domain is lost and usually results in severe inaccuracy of the error estimate and bound. Therefore, it is hardly an important issue to obtain the best possible constants in (27)-(29).

Although the exact constants are not known, the error indicators defined in (29) with approximate constants might be used for driving mesh adaptivity. Given the estimate $E_{h}$ for the error in the energy norm, the relative error is calculated from

$e_{\text {rel }}=\frac{\left\|e_{h}\right\|_{E}}{\|u\|_{E}} \approx \frac{E_{h}}{\left\|u_{h}\right\|_{E}}$

One strategy is to enrich the finite element space if the relative error $e_{\text {rel }}$ exceeds a specified tolerance, say $\gamma_{\text {tol }}=0.01$. The mesh is considered optimal when the contribution of each element to the total error is about the same so that the following ratio indicates if a single element is to be refined or not

$\xi_{K}=\frac{m \cdot \eta_{K}}{\gamma_{\text {tol }} \cdot\left\|u_{h}\right\|_{E}}= \begin{cases}>1 & \text { refine element } \\ <1 & \text { enlarge element } \\ =1 & \text { no change }\end{cases}$

where $m$ is the number of elements used. To avoid overrefinement due to overestimation, it is commonly used practice that per refinement step only some percentage of those elements which exceed the ratio $\xi_{K}>1$ is refined.

\subsection{Implicit error estimators}

Implicit error estimators involve the solution of auxiliary boundary value problems whose solution yields an approximation to the actual error. The interest in implicit schemes stems from the fact that in explicit schemes the whole information for the total error is obtained only from the given solution, when it might be possible to obtain more accurate information on the error by solving additional auxiliary problems.

The boundary value problems to be solved are local, which means that they are posed either on a small patch of elements (subdomain residual method) or even only on one single element (element residual method). In general, a drawback of the subdomain residual method can be that solving the local problems is rather expensive, since each element is considered several times. On the other hand, the element residual method needs to approximate the prescribed Neumann boundary data on each single element.

\subsubsection{Element residual method}

In the element residual method (see Bank and Weiser [15]), we define by $e_{K}=u-u_{h}$ the local error on a single element $K$ that satisfies for our model problem the variational problem

$a\left(e_{K}, v\right)_{K}=\int_{K} R v \mathrm{~d} \Omega+\int_{\partial K}\left(\frac{\partial u}{\partial n}-\frac{\partial u_{h}}{\partial n}\right) v \mathrm{~d} s \quad \forall v \in V_{K}$

In (32) the subscript $K$ denotes the restriction of the bilinear form to a single element where the trial and test space is defined by

$V_{K}=\left\{v \in H^{1}(K): v=0 \quad\right.$ on $\left.\quad \partial K \cap \Gamma_{\mathrm{D}}\right\}$

In order to impose the correct boundary conditions we have to check whether the boundary $\partial K$ of the single element intersects a portion of the boundary $\Gamma$ of the domain $\Omega$. On the Dirichlet part of the boundary the contribution to the local error is zero. Clearly, on the Neumann part of the global boundary, the true flux in (32) equals the prescribed data $g$. If the element boundary matches an interelement boundary, the true flux, of course, is unknown. Therefore, an approximation to the true flux is taken from the finite element solution

$\frac{\partial u}{\partial n} \approx \frac{\partial \bar{u}_{h}}{\partial n}=\frac{1}{2} \mathbf{n} \cdot\left(\nabla u_{h}+\nabla u_{h}^{\prime}\right)$

where $\nabla u_{h}^{\prime}$ denotes the gradient in element $K^{\prime}$ sharing a common edge with element $K$. Hence, the idea is to average the discontinuous finite element flux at interelement boundaries to find an approximation to the true flux. Then the local problem means to find a function $\zeta_{K} \in V_{K}$ that satisfies

$a\left(\zeta_{K}, v\right)_{K}=\int_{K} R v \mathrm{~d} \Omega+\int_{\partial K}\left(\frac{\partial \bar{u}_{h}}{\partial n}-\frac{\partial u_{h}}{\partial n}\right) v \mathrm{~d} s \quad \forall v \in V_{K}$

With the solutions $\zeta_{K}$ of the single elements known, the error is estimated using

$\left\|e_{h}\right\|_{E}^{2} \approx\left(E_{h}\right)^{2}=\sum_{K \in \mathscr{T}_{h}} \eta_{K}^{2} \quad$ with $\quad \eta_{K}^{2}=\left\|\zeta_{K}\right\|_{E}^{2}$ 
Unfortunately, the existence and uniqueness of the variational problem (35) is not guaranteed due to the possible incompatibility of the prescribed Neumann data. To overcome this drawback several techniques have been proposed. For instance, the problem is reformulated over a subspace $\tilde{V}_{K} \subset V_{K}$ for which the local bilinear form $a(., .)_{K}$ is elliptic. Another approach employs equilibrated boundary data so that the local problems remain well posed and therefore the consistency of the estimator is recovered (see e.g. Refs. [5,6]). It should be noted that, in this approach, an upper error bound can easily be obtained using the Cauchy-Schwarz inequality. However, the upper estimate of the error is only guaranteed if the local problems are computed exactly. If not, the estimator could underestimate the error [6], but in [16] it is shown that the error arising in the approximation of the local problems can be estimated with explicit estimation schemes.

\subsubsection{Subdomain residual method}

The basic idea of the subdomain residual method is to decompose the global residual Eq. (17) into a number of local problems on small element patches with homogeneous Dirichlet boundary conditions [9,11,17]. Recently, new versions of the subdomain residual method have been proposed which are more flexible in the choice of the boundary conditions (see Refs. [18-21]). In the approach of Prudhomme et al. [21], the subdomain residual method starts with the fundamental error representation (17) and utilizes the partition of unityproperty of the shape functions. Consider that in our model problem for a mesh of $n$ nodes, we use $n$ element patches, each node defining as its patch the elements coupling into the node. Let $\varphi_{i}(x)$ be the shape (or interpolation) function corresponding to node $i$. Then of course

$\sum_{i=1}^{n} \varphi_{i}(x)=1$

where we consider all boundary conditions removed. Inserting (37) into (17) leads to

$a\left(e_{h}, v\right)=\mathscr{R}_{h}\left(v \sum_{i=1}^{n} \varphi_{i}\right)=\sum_{i=1}^{n} \mathscr{R}_{h}\left(v \varphi_{i}\right) \quad \forall v \in V$

We introduce on each patch $\omega_{i}=\operatorname{supp} \varphi_{i}$ the "weighted" bilinear form

$a_{\varphi_{i}}(u, v)=\int_{\omega_{i}} \varphi_{i}(\nabla u \cdot \nabla v) \mathrm{d} \Omega$

associated with the norm $\|v\|_{\varphi_{i}}=\sqrt{a_{\varphi_{i}}(v, v)}$. Then on each patch $\omega_{i}$ the following local Neumann problems are considered:

Find $\zeta_{i} \in W$ such that

$a_{\varphi_{i}}\left(\zeta_{i}, \psi\right)=\mathscr{R}_{h}\left(\psi \varphi_{i}\right) \quad \forall \psi \in W$ where $W$ is a space of functions on $\omega_{i}$ defined by

$$
\begin{array}{r}
W=\left\{\psi \in H^{1}\left(\omega_{i}\right): \int_{\omega_{i}} \psi \varphi_{i} \mathrm{~d} \Omega=0\right. \\
\text { and } \left.\int_{\omega_{i}}|\nabla \psi|^{2} \varphi_{i} \mathrm{~d} \Omega<\infty\right\}
\end{array}
$$

for an interior node $i$ and a node $i$ located on the boundary $\Gamma_{\mathrm{N}}$ and

$$
\begin{gathered}
W=\left\{\psi \in H^{1}\left(\omega_{i}\right): \psi=0 \text { on } \partial \omega_{i} \cap \Gamma_{\mathrm{D}}\right. \\
\text { and } \left.\int_{\omega_{i}}|\nabla \psi|^{2} \varphi_{i} \mathrm{~d} \Omega<\infty\right\}
\end{gathered}
$$

for a node $i$ located on the boundary $\Gamma_{\mathrm{D}}$. The reason for this particular choice of the space $W$ is that rigid body modes are this way eliminated, otherwise the solution of some local problems in (40) would only be defined up to a constant. Clearly, after assembling the local problems we have

$a\left(e_{h}, v\right)=\sum_{i=1}^{n} \mathscr{R}_{h}\left(v \varphi_{i}\right)=\sum_{i=1}^{n} a_{\varphi_{i}}\left(\zeta_{i}, v\right) \quad \forall v \in V$

Taking $v=e_{h}$ and using the Cauchy-Schwarz inequality yields the guaranteed upper error bound

$\left\|e_{h}\right\|_{E}=\sqrt{a\left(e_{h}, e_{h}\right)} \leqslant \sqrt{\sum_{i=1}^{n} a_{\varphi_{i}}\left(\zeta_{i}, \zeta_{i}\right)}$

Of course, the exact solutions $\zeta_{i}$ of the local problems are unknown. Therefore, we seek approximations $\zeta_{i}^{h}$ on each patch yielding the upper error bound [21]

$\left\|e_{h}\right\|_{E} \leqslant \sqrt{\sum_{i=1}^{n} a_{\varphi_{i}}\left(\zeta_{i}^{h}, \zeta_{i}^{h}\right)}+2 \inf _{v \in V^{p+q}}\|u-v\|_{E} \quad \forall v \in V^{p+q}$

where $p$ is the degree of the polynomials used in the approximation of the original problem and $q$ is the additional degree of the polynomials used for solving the discrete local problems. We note that for $q=0$ this error estimator is not sharp since it doubles the bound which is already provided by the optimality condition. However, the second term on the right-hand side of (45) should decrease as the polynomial degree used to solve the local problems increases. In practice we might simply employ

$E_{h}=\sqrt{\sum_{i=1}^{n} a_{\varphi_{i}}\left(\zeta_{i}^{h}, \zeta_{i}^{h}\right)}$

Using the approximations $\zeta_{i}^{h}$, it is also possible to obtain a lower bound on the error (see Ref. [21]).

The essential feature of this error estimator is that it localizes both the residual and the bilinear form, and therefore allows to pose Neumann boundary conditions for the local problems on interior patches. The effect of the flux jumps between elements is implicitly taken into 
account since the local problems on each patch necessarily include the interelement edges. The error estimator is free of any constants and does not require flux equilibration. In [21] the error estimator was tested in some numerical experiments. However, for practical analysis, the error estimate established in (46) is, of course, expensive to compute.

\subsection{Recovery-based error estimators}

Recovery-based error estimators make use of the fact that the gradient of the finite element solution is in general discontinuous across the interelement boundaries. Of course, a simple way to visualize the error across the element boundaries is to use iso-bands of stresses as proposed by Sussman and Bathe [22], see also [1], which are compared with smoothed values. Here, the underlying idea is to post-process the gradient and to find an estimate for the true error by comparing the post-processed gradient and the nonpost-processed gradient of the approximation. In particular, let $\mathscr{M}\left[u_{h}\right]$ denote an "improved" approximation to the gradient, then the a posteriori error estimator is taken to be

$\left(E_{h}\right)^{2}=\int_{\Omega}\left|\mathscr{M}\left[u_{h}\right]-\nabla u_{h}\right|^{2} \mathrm{~d} \Omega$

It is remarkable that this rather heuristic approach gives surprisingly good results. Under suitable conditions, the post-processed gradient $\mathscr{M}\left[u_{h}\right]$ is labeled superconvergent because the finite element non post-processed approximation is closer to the smoothed gradient than to the exact gradient. This means that for linear interpolation functions we have

$d_{1}=\left\|\mathscr{M}\left[u_{h}\right]-\nabla u_{h}\right\|_{L^{2}(\Omega)} \leqslant c h^{2}$

whereas the distance between the exact gradient and the finite element gradient behaves like

$d_{2}=\left\|\nabla u-\nabla u_{h}\right\|_{L^{2}(\Omega)} \leqslant c h$

But there are situations in which this insight is not of practical value. Suppose that the finite element gradient is an inaccurate approximation of the exact gradient, then the "superconvergence" relation $d_{1}<d_{2}$ does not help to estimate the true error (see Fig. 2). ${ }^{2}$

A much researched recovery-based error estimator was proposed by Zienkiewicz and Zhu [23], who suggested to post-process the discontinuous gradient in terms of the interpolation functions $\varphi_{i} \in V_{h}$ as follows

\footnotetext{
${ }^{2}$ In this context, the attribute "superconvergent" is adopted from the literature, but it is somewhat misleading since it does not provide any information about the distance between the exact solution and the post-processed finite element approximation.
}

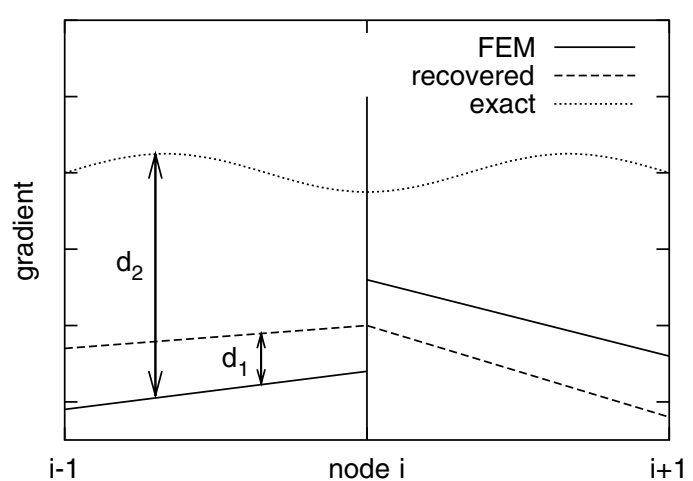

Fig. 2. One-dimensional schematic example for the superconvergence property of the recovered gradient.

$\nabla u_{h}^{\star}=\sum_{i=1}^{n}\left(\nabla u_{h}^{\star}\right)_{i} \varphi_{i}$

Here, the unknown nodal values $\left(\nabla u_{h}^{\star}\right)_{i}$ are determined by a standard $L^{2}$-projection

$\int_{\Omega} \varphi_{j}\left(\nabla u_{h}^{\star}-\nabla u_{h}\right) \mathrm{d} \Omega=0 \quad j=1, \ldots, n$

This projection results in a linear system for determining the nodal values

$\sum_{i=1}^{n} \int_{\Omega} \varphi_{j} \varphi_{i} \mathrm{~d} \Omega\left(\nabla u_{h}^{\star}\right)_{i}=\int_{\Omega} \varphi_{j} \nabla u_{h} \mathrm{~d} \Omega \quad j=1, \ldots, n$

which is often solved only approximately by diagonalizing the coefficient matrix. Then, to obtain an error estimator, the improved gradient $\nabla u_{h}^{\star}$ is used instead of the true gradient

$\left\|e_{h}\right\|_{E}^{2} \approx a\left(e_{h}^{\star}, e_{h}^{\star}\right)=\int_{\Omega}\left(\nabla u_{h}^{\star}-\nabla u_{h}\right)^{2} \mathrm{~d} \Omega$

In practical analysis the error estimate is calculated elementwise

$$
\begin{aligned}
& \left\|e_{h}\right\|_{E}^{2} \approx\left(E_{h}\right)^{2}=\sum_{K \in \mathscr{T}_{h}} \eta_{K}^{2} \\
& \text { with } \eta_{K}^{2}=\left\|\nabla u_{h}^{\star}-\nabla u_{h}\right\|_{L^{2}(K)}^{2}
\end{aligned}
$$

This error representation is clearly close to the procedure proposed by Sussman and Bathe [22].

The crucial questions are whether the improved gradients provide a better solution than the originally calculated gradients and whether the error estimate can be used to obtain a bound on the true error. Carstensen and Funken [24] have shown that under certain smoothness conditions this error estimate is asymptotically exact up to higher order terms. Numerical examples usually show that this error estimator is effective in smooth problems when using interpolation functions of degree $p=1$. 
Refs. [25,26] show that the approach of Zienkiewicz and Zhu can lead to improved estimates if the interpolation is not using the nodes but so-called "superconvergent points" in element patches of the domain. This approach is called the superconvergent patch recovery technique. Actually, in [6] it is shown that sometimes superconvergence can also be obtained without using superconvergent points. Further improvements of recovery-based methods can be found in [27] and an analysis of these methods for use on unstructured meshes is presented in [28].

However, of course, the Zienkiewicz-Zhu algorithm is not effective in the presence of material discontinuities since in a patch-based algorithm these effects are smoothed out. Therefore, Hiller and Bathe [29] propose an element-based error estimator that uses higherorder-accuracy points to recover the strain field. For one-dimensional test cases this error estimator is found to be highly accurate compared to the superconvergent patch recovery technique. However, the extension of this approach to $2 \mathrm{D}$ and $3 \mathrm{D}$-problems is not obvious.

Another weak point of the Zienkiewicz-Zhu algorithm is the implicit assumption that oscillations indicate errors and that smooth stresses mean accurate stresses. Hence, the method might break down if the non postprocessed stresses are smooth. In the following problem [5]

$$
-E A u^{\prime \prime}(x)=\mu \sin \left(2^{m} \pi x\right) ; \quad u(0)=u(1)=0 ; \quad m>0
$$

a bar is stretched and compressed by an oscillating longitudinal force with an amplitude $\mu$. Suppose this problem is solved on a uniform mesh of $2^{n}$ linear elements $(n \leqslant m)$ with the nodes located at the points

$x_{k}=k / 2^{n} ; \quad k=0,1, \ldots, 2^{n}$

Then the piecewise linear finite element solution is zero, because we know that it interpolates the exact solution at the nodes where the exact solution is zero. For example, let $E A=1$, then we have

$u(x)=\frac{\mu}{4^{m} \pi^{2}} \sin \left(2^{m} \pi x\right)$

Hence, the finite element normal forces as well as the recovered normal forces are zero and infinitely smooth and the Zienkiewicz-Zhu error estimator predicts zero error, while in truth the exact normal force is far from zero and oscillates as shown in Fig. 3. On the other hand, the error estimator of Hiller and Bathe [29] yields a maximal amplitude of the normal force equal to $15.2789 \mathrm{kN}$ for the choice $N^{\prime}=2$ and $12.4528 \mathrm{kN}$ for $N^{\prime}=4$ compared to the exact value of $12.5664 \mathrm{kN}$, see Ref. [29] for the definition of $N^{\prime}$. Hence, using the estimator of Ref. [29] the error is correctly detected.

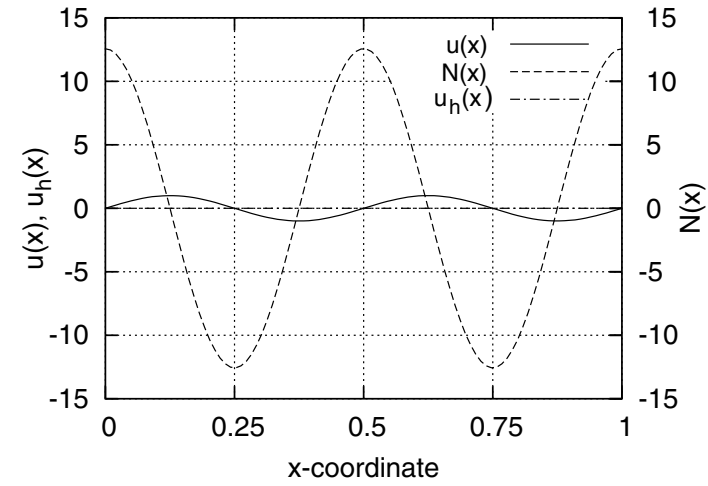

Fig. 3. Oscillating longitudinal displacements $u(x)$ produce an oscillating normal force $N(x)$ but the finite element solution $u_{h}(x)$ is zero.

\subsection{Concluding remarks}

In order to assess the presented techniques, we have to realize that there are two different goals using error estimation procedures. The first goal is to estimate the actual error in a suitable norm and ideally provide useful actual error bounds. Note that upper and lower error bounds give of course always an error estimate which is, however, not very useful if the difference in the bounds is too large. An effective error estimate assesses the true error accurately but may not give a guaranteed error bound. Actually, all the above mentioned error bounds when evaluated in general analyses are not guaranteed and must therefore be used with caution.

The second goal is to steer an adaptive scheme to obtain meshes which are optimal with respect to the aim of the computation. Then - while the re-meshing or refinement algorithms can be expensive - the error estimation need not to be very accurate and inexpensive, explicit schemes with approximate constants can be used to provide criteria for the adaptive mesh refinement. Recovery-based error estimators are inexpensive too and also provide quite useful error estimates for this purpose. While there is of course no need to use an actual error bound to establish improved and refined meshes, it is clear that in the final step of mesh and solution acceptance an actual error bound is also desired.

From a practical point of view, recovery-based error estimators are efficient compared to other methods and can be generally used. Since all the error estimators do not provide in general guaranteed bounds, the estimation technique most useful is probably the method that works efficiently in general analyses (including nonlinear analyses) and for general finite element discretizations, and provides sufficient or reasonable accuracy in the error estimation. 


\section{Goal-oriented error estimates}

In finite element analysis it is frequently the case that the analyst is more interested in certain output data of the finite element approximation than in the global energy norm. In order to find an estimate for the error in the output data pertaining to a specific quantity, or to find at least an effective mesh to accurately solve for this quantity, error estimators for the energy norm are not useful. Hence, more recently so-called goal-oriented error estimates were developed, which estimate the error in individual quantities of interest using duality techniques [30-41]. Let $Q(u)$ denote such a (linear) quantity of interest, as for example the mean value of the $x$-component of the gradient in our model problem over a (not necessarily) small patch $\Omega_{\varepsilon}$,

$Q(u)=\left|\Omega_{\varepsilon}\right|^{-1} \int_{\Omega_{\varepsilon}} \nabla_{x} u \mathrm{~d} \Omega$

The key for estimating the error in such quantities is the formulation of an auxiliary problem, which is the dual problem to the primal problem actually considered, and which filters out the necessary information for an accurate estimate for the error in the quantity of interest. In the following we recall the basic framework of these procedures and then briefly discuss specific applications using this framework.

\subsection{The basic framework}

In order to develop a general framework, it is convenient to regard the quantity of interest as a bounded, linear functional $Q: V \rightarrow \mathbb{R}$ in the dual space $V^{\prime}$ associated with the norm

$\|Q\|_{V^{\prime}}=\sup _{v \in V} \frac{|Q(v)|}{\|v\|_{V}}$

Of course, for the bilinear form considered in our model problem, we have a unique $z$ which is the solution of the variational problem

$a(z, v)=Q(v) \quad \forall v \in V$

The solution of this problem is referred to as the dual solution and can be interpreted as the generalized Green's function, or the influence function, related to the functional $Q(v)$.

The objective in the following is to find an estimate for the error

$Q\left(e_{h}\right)=Q(u)-Q\left(u_{h}\right)$

where $Q\left(u_{h}\right)$ denotes the finite element approximation of the quantity of interest and $Q(u)$ is the exact value. Setting $v=e_{h}$ in (60) gives the exact error representation

$Q\left(e_{h}\right)=a\left(z, e_{h}\right)=l(z)-a\left(u_{h}, z\right)=\mathscr{R}_{h}(z)$
Employing the Galerkin orthogonality condition (10) with $v_{h}=z_{h}$ yields the starting point for goal-oriented error estimators

$Q\left(e_{h}\right)=a\left(z-z_{h}, e_{h}\right)=\mathscr{R}_{h}\left(z-z_{h}\right)$

In (63) the finite element approximation $z_{h}$ of the dual solution is obtained by solving the discrete problem

$a\left(z_{h}, v_{h}\right)=Q\left(v_{h}\right) \quad \forall v_{h} \in V_{h} \subset V$

Before focusing on the error estimation part in detail, there are three points that we want to mention. Firstly, to solve the discrete dual problem (64), we have to apply equivalent nodal forces $f_{i}$ defined by the right-hand side of (64) and the definition of the functional as in (58). Thus, for (58), for a certain interpolation function $\varphi_{i} \in V_{h}$ we have

$f_{i}=Q\left(\varphi_{i}\right)=\left|\Omega_{\varepsilon}\right|^{-1} \int_{\Omega_{\varepsilon}} \nabla_{x} \varphi_{i} \mathrm{~d} \Omega$

Secondly, using that the finite element spaces for the primal problem and the dual problem are the same, we have

$$
\begin{aligned}
Q\left(u_{h}\right) & =a\left(z_{h}, u_{h}\right)=a\left(u_{h}, z_{h}\right)=l\left(z_{h}\right) \\
& =\int_{\Omega} f z_{h} \mathrm{~d} \Omega+\int_{\Gamma_{N}} g z_{h} \mathrm{~d} s
\end{aligned}
$$

which means that $Q\left(u_{h}\right)$ can be evaluated by forming the scalar product between the data $f$ and $g$ of the primal problem and the finite element solution $z_{h}$ of the dual problem [42]. We note that formula (66) expresses a fundamental symmetry condition, since there are always two ways to calculate the quantity of interest $Q\left(u_{h}\right)$ :

1. Calculate the finite element solution $u_{h}$ as usual; postprocess the gradient by differentiation of the shape functions and perform the necessary integrations.

2. Calculate the influence function $z_{h}$ and form the scalar product between the data of the primal problem and $z_{h}$.

Clearly, the more accurate the approximation $z_{h}$ for the influence function $z$, the more accurate is the calculation of the quantity of interest itself. Note that the well-known "unit-dummy-load" method in structural mechanics is a special case of Eq. (66). Furthermore, the use of (66) in many mixed finite element methods is direct since (66) only uses the symmetry of the bilinear form.

Thirdly, it should be pointed out that the dual approach is very general in that even global quantities like the mean value of the solution can be defined as a quantity of interest

$Q(u)=|\Omega|^{-1} \int_{\Omega} u \mathrm{~d} \Omega$ 
which yields the following variational problem seeking the dual solution

$a(z, v)=Q(v)=|\Omega|^{-1}(1, v) \quad \forall v \in V$

Hence, the solution of the dual problem equals the solution of the primal problem for a constant domain load $f=1$ up to the factor $|\Omega|^{-1}$.

\subsection{Specific applications using the basic framework}

In the following, we discuss briefly specific applications using the basic framework presented in Section 5.1 in order to estimate the error in a quantity of interest.

\subsubsection{Energy norm based estimates}

There are several strategies for goal-oriented error estimation based on energy norm estimates of the primal problem and the dual problem. For instance, using the error representation (63) and employing the CauchySchwarz inequality, the following upper error bound is obtained

$$
\left|Q\left(e_{h}\right)\right|=\left|a\left(z-z_{h}, u-u_{h}\right)\right| \leqslant\left\|z-z_{h}\right\|_{E}\left\|u-u_{h}\right\|_{E}
$$

Hence, the error in the quantity of interest is bounded by the error in the energy norm of the primal problem weighted with the error in the energy norm of the dual problem. It follows that any of the error estimators derived in Section 4 may be used to estimate the error in the evaluation of the quantity of interest. Moreover, the estimate (69) shows that the convergence rate of $\left|Q\left(e_{h}\right)\right|$ is larger than the convergence rate of the error in the energy norm, which means that any linear functional acting on the finite element space is superconvergent compared to the convergence in the energy norm. Of course, the actual convergence rate of the functional depends on the regularity of the solutions of the primal problem and the dual problem.

In practice, the Cauchy-Schwarz inequality is applied elementwise in (69), which leads to

$\left|Q\left(e_{h}\right)\right| \leqslant \sum_{K \in \mathscr{T}_{h}}\left\{\left\|z-z_{h}\right\|_{E(K)}\left\|u-u_{h}\right\|_{E(K)}\right\}$

However, goal-oriented error estimators based on energy norm estimates as in (69) or (70) eliminate the cancellation of errors over the domain, and therefore produce significant error overestimation in general. In [43] it is shown that this overestimation gets even worse by increasing the polynomial degree of the interpolation functions used in a p-version of the finite element method.

Different techniques with the aim to provide tighter error bounds have been proposed. For instance, Prudhomme and Oden $[44,45]$ employ the parallelogram identity to problems with symmetric bilinear forms leading to upper and lower bounds on the error. In this ap- proach, using $\varepsilon_{h}=z-z_{h}$, the starting point is the error representation (see also [46])

$Q\left(e_{h}\right)=a\left(e_{h}, \varepsilon_{h}\right)=\frac{1}{4}\left\|s e_{h}+\frac{\varepsilon_{h}}{s}\right\|_{E}^{2}-\frac{1}{4}\left\|s e_{h}-\frac{\varepsilon_{h}}{s}\right\|_{E}^{2}$

where $s \in \mathbb{R}$ is a scaling factor chosen to be

$s=\sqrt{\frac{\left\|\varepsilon_{h}\right\|_{E}}{\left\|e_{h}\right\|_{E}}}$

Let $\eta_{\text {low }}^{+}, \eta_{\text {upp }}^{+}, \eta_{\text {low }}^{-}, \eta_{\text {upp }}^{-}$denote error estimators that satisfy

$\eta_{\text {low }}^{+} \leqslant\left\|s e_{h}+\frac{\varepsilon_{h}}{s}\right\|_{E} \leqslant \eta_{\text {upp }}^{+}$

$\eta_{\text {low }}^{-} \leqslant\left\|s e_{h}-\frac{\varepsilon_{h}}{s}\right\|_{E} \leqslant \eta_{\text {upp }}^{-}$

then (71) implies

$$
\begin{aligned}
\frac{1}{4}\left(\eta_{\text {low }}^{+}\right)^{2}-\frac{1}{4}\left(\eta_{\text {upp }}^{-}\right)^{2} & \leqslant Q\left(e_{h}\right) \\
& \leqslant \frac{1}{4}\left(\eta_{\text {upp }}^{+}\right)^{2}-\frac{1}{4}\left(\eta_{\text {low }}^{-}\right)^{2}
\end{aligned}
$$

which provides lower and upper bounds for the error $Q\left(e_{h}\right)$. In [45] these error bounds have numerically been found to be accurate for simple model problems when the error estimators defined in (73) and (74) are calculated using implicit methods.

In this context, Stein and Ohnimus [47] note that by neglecting the lower error bound $\eta_{\text {low }}^{-}$, the upper error bound in (75) reduces to (69), which demonstrates that (75) gives a better error bound than (69). Hence, the lower bound error estimation is essential to obtain an efficient upper error bound for goal-oriented error estimation. Of course, (75) is also providing better error bounds than

$-\frac{1}{4}\left(\eta_{\text {upp }}^{-}\right)^{2} \leqslant Q\left(e_{h}\right) \leqslant \frac{1}{4}\left(\eta_{\text {upp }}^{+}\right)^{2}$

but these bounds are less expensive to evaluate [37,44].

To steer an adaptive refinement process using energy norm based estimates, we can use the upper error bound defined in (70) in combination with explicit error estimators for the energy norm error yielding

$\left|Q\left(e_{h}\right)\right| \leqslant \eta_{h}=\sum_{K \in \mathscr{T}_{h}} \omega_{K} \eta_{K}$

where the local contributions to the error can be calculated from

$\omega_{K}=\left(c_{1} h_{K}^{2}\left\|R_{z}\right\|_{L^{2}(K)}^{2}+c_{2} h_{K}\left\|J_{z}\right\|_{L^{2}(\partial K)}^{2}\right)^{1 / 2}$

$\eta_{K}=\left(c_{1} h_{K}^{2}\|R\|_{L^{2}(K)}^{2}+c_{2} h_{K}\|J\|_{L^{2}(\partial K)}^{2}\right)^{1 / 2}$

In (78) and (79) $c_{1}, c_{2}$ are the unknown constants mentioned already with (29) and the $R_{z}$ and $J_{z}$ denote the 
element residuals and jumps of the gradient corresponding to the dual problem. Given the estimate for the quantity of interest based on (77), or any other goal-oriented error estimator, the relative error in the goal quantity is calculated from

$e_{\text {rel }}=\frac{\left|Q\left(e_{h}\right)\right|}{|Q(u)|} \approx \frac{\eta_{h}}{\left|Q\left(u_{h}\right)\right|}$

As in the case of error estimates for the energy norm, one strategy is to enrich the finite element space if the relative error $e_{\text {rel }}$ exceeds a specified tolerance $\gamma_{\text {tol. }}$. For equal distribution of errors across elements, the following ratio is used to indicate whether a single element is to be refined or not

$\xi_{K}=\frac{m \cdot\left(\omega_{K} \eta_{K}\right)}{\gamma_{\mathrm{tol}} \cdot\left|Q\left(u_{h}\right)\right|}= \begin{cases}>1 & \text { refine element } \\ <1 & \text { enlarge element } \\ =1 & \text { no change }\end{cases}$

Since we are considering the error bound in (70) and hence overrefinement due to overestimation is still an issue, in practice, it may be best if per refinement step only some percentage of those elements which exceed the ratio $\xi_{K}>1$ is refined. In order to assess the accuracy of the error estimate for the quantity of interest, we use the effectivity index

$\lambda=\frac{\eta_{h}}{\left|Q\left(e_{h}\right)\right|}$

\subsubsection{The dual-weighted residual method}

Rannacher and co-workers (see [48] and the references therein), proposed the dual-weighted residual method, which is based on an exact representation of the error in the quantity of interest as follows

$$
\begin{aligned}
Q\left(e_{h}\right) & =a\left(e_{h}, z-v_{h}\right) \\
& =\sum_{K \in \mathscr{T}_{h}}\left\{\int_{K} R\left(z-v_{h}\right) \mathrm{d} \Omega+\int_{\partial K} J\left(z-v_{h}\right) \mathrm{d} s\right\}
\end{aligned}
$$

where $R$ and $J$ are the element residuals and jumps of the primal problem defined in (20) and (21), respectively ${ }^{3}$ and $v_{h} \in V_{h}$. To drive an adaptive mesh refinement process, the following element error indicators can be used

$\eta_{K}=\left|\left(R, z-v_{h}\right)_{K}+\left(J, z-v_{h}\right)_{\partial K}\right|$

which yield the guaranteed upper error bound

$\left|Q\left(e_{h}\right)\right| \leqslant \sum_{K \in \mathscr{T}_{h}} \eta_{K}$

\footnotetext{
${ }^{3}$ In (83) we assume that on interelement boundaries $\partial K \nsubseteq \Gamma$ the jumps $J$ are multiplied by $1 / 2$ to distribute the error equally onto the two elements sharing the common edge.
}

There are several strategies for evaluating the unknown dual solution in (83). One possibility is to solve the dual problem by using a higher-order method where, for instance, biquadratic functions are used instead of bilinear functions. This yields the approximate error representation

$$
\begin{aligned}
Q\left(e_{h}\right) \approx & \sum_{K \in \mathscr{T}_{h}}\left\{\int_{K} R\left(z_{h}^{(2)}-\mathscr{I}_{h} z_{h}^{(2)}\right) \mathrm{d} \Omega\right. \\
& \left.+\int_{\partial K} J\left(z_{h}^{(2)}-\mathscr{I}_{h} z_{h}^{(2)}\right) \mathrm{d} s\right\}
\end{aligned}
$$

where the upper index (2) denotes the solution obtained with a higher-order method and $\mathscr{I}_{h}$ is the interpolant on $V_{h}$. Instead of using this rather expensive approach, another technique consists of approximating the dual solution by employing higher-order interpolation functions defined over single patches of the domain, resulting in the error representation

$Q\left(e_{h}\right) \approx \sum_{K \in \mathscr{T}_{h}}\left\{\int_{K} R\left(\mathscr{I}_{2 h} z_{h}-z_{h}\right) \mathrm{d} \Omega+\int_{\partial K} J\left(\mathscr{I}_{2 h} z_{h}-z_{h}\right) \mathrm{d} s\right\}$

where $\mathscr{I}_{2 h}$ denotes the interpolant obtained with higherorder interpolation functions. Both strategies lead to useful error indicators and can even lead to effectivity indices close to 1.0 depending on the problem considered. However, error estimates using (86) or (87) may underestimate the actual error since the dual problem is only solved approximately. In contrast, error bounds based on (85) may significantly overestimate the true error, since all the error indicators contribute positive terms to the estimate.

Note that instead of applying integration by parts to obtain the element residuals and jump terms in (83), we could also leave the residual in weak form yielding for our model problem the error representation

$Q\left(e_{h}\right)=\sum_{K \in \mathscr{T}_{h}}\left\{\int_{K} l\left(z-v_{h}\right) \mathrm{d} \Omega-\int_{K} \nabla u_{h} \cdot \nabla\left(z-v_{h}\right) \mathrm{d} \Omega\right\}$

Compared to (83), error estimates based on (88) are accurate as well and the derivation of local error indicators according to (84) is straightforward.

The dual-weighted residual method has been successfully applied in the solution of various problem, however the extension to nonstandard problems such as fluidstructure interactions is still to be achieved.

\subsubsection{Direct use of influence functions}

Instead of calculating element-based residuals, we can also directly employ influence functions and (66). This approach was used by Grätsch and Bathe in shell analyses [49]. Using a continuum mechanics shell formulation $[1,50]$, the error in a linear quantity of interest can be written as 
$Q\left(\mathbf{E}_{h}\right)=\int_{\Omega_{\mathrm{ref}}} \mathbf{F} \cdot \mathbf{Z}_{\mathrm{ref}} \mathrm{d} V-\int_{\Omega_{h}} \mathbf{F} \cdot \mathbf{Z}_{h} \mathrm{~d} V$

where $\mathbf{E}_{h}$ denotes the error in the shell finite element approximation of the primal problem, $\mathbf{Z}_{\mathrm{ref}}$ is the exact influence function corresponding to a $3 \mathrm{D}$ reference domain $\Omega_{\text {ref }}, \mathbf{Z}_{h}$ denotes the finite element solution for $\mathbf{Z}_{\text {ref }}$ obtained on a 3D computational domain $\Omega_{h}$, and $\mathbf{F}$ are body loads. Assuming $\mathbf{Z}_{h}$ is a MITC4 shell finite element solution [51,52], a useful strategy is to replace the unknown dual solution with an approximation $\mathbf{Z}_{h}^{\text {(MITC9) }}$ corresponding to a MITC9 shell finite element formulation such that

$Q\left(\mathbf{E}_{h}\right) \approx \int_{\Omega_{h 9}} \mathbf{F} \cdot \mathbf{Z}_{h}^{(\mathrm{MITC} 9)} \mathrm{d} V-\int_{\Omega_{h}} \mathbf{F} \cdot \mathbf{Z}_{h} \mathrm{~d} V$

where $\Omega_{h 9}$ denotes the 3D reference mesh using MITC9 elements. In [49] it is found in some numerical studies that the error representation (90) leads to accurate error estimates and to effectivity indices close to 1.0 (since (90) takes into account the cancellation effect of the error over the domain). The error estimate includes the error due to the approximation in the geometry of the shell structure.

For the purpose of driving mesh adaptivity we can also obtain local error indicators by considering

$Q\left(\mathbf{E}_{h}\right) \approx \sum_{K \in \mathscr{T}_{h}}\left\{\int_{K} \mathbf{F} \cdot \mathbf{Z}_{h}^{(\mathrm{MITC} 9)} \mathrm{d} V-A_{h}\left(\mathbf{Z}_{h}, \mathbf{U}_{h}\right)_{K}\right\}$

in which $A_{h}(.,$.$) denotes the bilinear form of the MITC4$ shell variational problem and $\mathbf{U}_{h}$ is the finite element solution. An example using the error representation (90) is given in Section 9.1.

\subsubsection{The Green's function decomposition method}

An important step in the use of goal-oriented error estimates is to seek an accurate solution of the dual problem. Considering individual output data such as point values, the presented procedures face the problem that in $H^{1}(\Omega)$ functions are not continuous (i.e. have meaningful point values) in two or three dimensions. Although an approximation of the Green's function can still be obtained, this problem can be circumvented either by using regularized functionals, as proposed for example in [44] or [48], or, when available, using analytical functions (see Kelly et al. $[53,54]$ ) or Grätsch and Hartmann [55]. The idea is to approximate the Green's function by splitting it into a regular part and a wellknown fundamental solution, and for this reason the method can be referred to as the Green's function decomposition method. Hence, the method can only be used for the rather restricted case where the analytical fundamental solution is available. Fundamental solutions in finite element discretizations have of course been used in various applications, and notably in fracture mechanics.
Consider our model problem (see Section 3) and let $u(\mathbf{x})$ denote the point quantity of interest at the point $\mathbf{x} \in \Omega$. Then using the corresponding Green's function and the problem data we have

$u(\mathbf{x})=\int_{\Omega} G_{0}(\mathbf{y}, \mathbf{x}) f(\mathbf{y}) \mathrm{d} \Omega+\int_{\Gamma_{\mathrm{N}}} G_{0}(\mathbf{y}, \mathbf{x}) g(\mathbf{y}) \mathrm{d} s$

where $G_{0}(\mathbf{y}, \mathbf{x})$ denotes the Green's function, which in this case is the displacement at the point $\mathbf{x}$ due to a point load at the point $\mathbf{y}$. The next step is to decompose the Green's function into a regular part $u_{\mathrm{R}}(\mathbf{y}, \mathbf{x})$ and the corresponding fundamental solution $F_{0}(\mathbf{y}, \mathbf{x})$

$G_{0}(\mathbf{y}, \mathbf{x})=u_{\mathrm{R}}(\mathbf{y}, \mathbf{x})+F_{0}(\mathbf{y}, \mathbf{x})$

where the fundamental solution is given by

$F_{0}(\mathbf{y}, \mathbf{x})=-\frac{1}{2 \pi} \ln r \quad$ with $\quad r=|\mathbf{y}-\mathbf{x}|$

The regular part is the solution of the following boundary value problem

$-\Delta u_{\mathrm{R}}=0 \quad$ in $\Omega ; \quad u_{\mathrm{R}}=-F_{0} \quad$ on $\Gamma_{\mathrm{D}} ;$

$g_{\mathrm{R}}=-\mathbf{n} \cdot \nabla F_{0}$ on $\Gamma_{\mathrm{N}}$

Hence the boundary conditions to obtain $u_{\mathrm{R}}$ are given by (the negative of) the fundamental solution on the boundary. The finite element approximation $u_{\mathrm{R}}^{h}$ to $u_{\mathrm{R}}$ is obtained by solving:

Find $u_{\mathrm{R}}^{h} \in V_{h}$ such that

$a\left(u_{\mathrm{R}}^{h}, v_{h}\right)=l\left(v_{h}\right) \quad \forall v_{h} \in V_{0, h}$

$u_{\mathrm{R}}^{h}=-F_{0} \quad$ on $\Gamma_{\mathrm{D}}$

where $V_{0, h}=H_{0}^{1}(\Omega) \cap V_{h}(\Omega)$ and the linear functional is defined by

$l\left(v_{h}\right)=-\int_{\Gamma_{\mathrm{N}}} g_{R} v_{h} \mathrm{~d} s$

Denoting the so-constructed Green's function by $G_{0, h}(\mathbf{y}, \mathbf{x})=u_{\mathrm{R}}^{h}(\mathbf{y}, \mathbf{x})+F_{0}(\mathbf{y}, \mathbf{x})$, the integral representation of the improved displacement becomes

$u_{h}(\mathbf{x})=\int_{\Omega} G_{0, h}(\mathbf{y}, \mathbf{x}) f(\mathbf{y}) \mathrm{d} \Omega+\int_{\Gamma_{\mathrm{N}}} G_{0, h}(\mathbf{y}, \mathbf{x}) g(\mathbf{y}) \mathrm{d} s$

To derive an a posteriori upper error bound for the improved quantity we proceed as follows. Let $e_{h}(-$ $\mathbf{x})=u(\mathbf{x})-u_{h}(\mathbf{x})$ denote the local error. Then we have by subtracting (99) from (92)

$e_{h}(\mathbf{x})=\int_{\Omega}\left(G_{0}-G_{0, h}\right) f \mathrm{~d} \Omega+\int_{\Gamma_{\mathrm{N}}}\left(G_{0}-G_{0, h}\right) g \mathrm{~d} s$

Since the exact Green's function $G_{0}$ and the approximated Green's function $G_{0, h}$ both contain the same fundamental solution we have as well 


$$
\begin{aligned}
e_{h}(\mathbf{x}) & =\int_{\Omega}\left(u_{\mathrm{R}}-u_{\mathrm{R}}^{h}\right) f \mathrm{~d} \Omega+\int_{\Gamma_{\mathrm{N}}}\left(u_{\mathrm{R}}-u_{\mathrm{R}}^{h}\right) g \mathrm{~d} s \\
& =a\left(u_{\mathrm{R}}-u_{\mathrm{R}}^{h}, u\right)
\end{aligned}
$$

Using the Galerkin orthogonality to introduce the finite element solution and applying the Cauchy-Schwarz inequality as usual yields the error bound

$\left|e_{h}(\mathbf{x})\right| \leqslant\left\|u_{\mathrm{R}}-u_{\mathrm{R}}^{h}\right\|_{E}\left\|u-u_{h}\right\|_{E}$

which states that the pointwise error is bounded by the error in the energy norm of the primal problem weighted with the error in the energy norm of the corresponding regular part problem. Comparing this result with (69), we realize that, to use (102), we can employ the usual error estimation techniques of goal-oriented error estimates since the error bounds in (69) and (102) only differ by the weighting factors. Clearly, the method is also closely related to the dual-weighted residual technique. The only difference is of course that in the Green's function decomposition method we actually construct an approximation of the Green's function for evaluating the local quantity with this function, while in other goal-oriented error estimates we simply differentiate the shape functions. Of course, the method can also be used for pointwise stresses; the only difference is that appropriate fundamental solutions need to be employed, which we can expect to yield high solution accuracy.

Indeed, in some numerical experiments the Green's function decomposition method gave excellent results in that high convergence rates in the required solutions were observed (see Ref. [55], and also Section 9.3). But, as mentioned already, the method is rather restrictive in that the fundamental solution needs to be available (see (97) and (98)). Additional considerations arise also, for example, if the load is not smooth, the material is not homogeneous [56], or the quantity of interest is a point value on the boundary [53].

\subsubsection{Exact-bounds approach}

The "exact-bounds approach" proposed by Peraire and co-workers [57] can be used to obtain guaranteed upper and lower bounds for quantities of interest. The basic idea in this approach is to use a displacementbased finite element method to obtain a guaranteed lower bound on the exact strain energy and to employ a hybrid (stress-based) finite element method using complementary energy principles to obtain a guaranteed upper bound. Recasting the problem in terms of the dual problem corresponding to the functional of interest, guaranteed bounds can be obtained for the quantity of interest using the solutions of local problems.

In contrast to other techniques presented in this paper, this approach is fundamentally different since it provides results to actually bound the true error in the finite element solution. However, from a practical point of view it is noted that in this approach the primal prob- lem and the dual problem have to be solved two times each, namely for the displacement method and the stress method. In addition, local problems have to be analyzed to obtain the error bounds, which could make the procedure costly in actual applications. Also, for some types of problems, notably (almost) incompressible linear and nonlinear problems and shell problems, mixed (or hybrid) formulations must be used to circumvent the "locking" phenomenon [1], and any approach to estimate errors needs to take this fact into account. So far the method has been presented for coercive linear model problems including (compressible) elasticity and advection-diffusion-reaction problems [58].

\subsubsection{Reduced-basis output bounds approach}

We also want to mention some achievements which do not exactly fit into the basic framework of Section 5.1 but which also provide estimates for quantities of interest.

The "reduced-basis output bounds approach" proposed by Patera and co-workers [59] addresses the practical case in which the quantity of interest needs to be computed for a certain number of parameters that are the values of specific variables describing some input data such as physical properties or geometry data.

The underlying idea of this approach is to solve the problem for a sample of the parameters on a very fine finite element mesh. Then, having these solutions, the solution corresponding to any other configuration within the parameter set is obtained by some interpolation technique. In other words, the unknown solution is expressed as a linear combination of the basis functions of a reduced-basis approximation space, which are the finite element solutions of the sample problems.

In practice, we generally do not know how to choose the size of the parameter space and it certainly depends on the desired accuracy, the selected quantities of interest, and the particular problem analyzed. In order to use a parameter space that is neither inefficient nor unacceptably uncertain, a posteriori error bounds for the quantity of interest are computed which, e.g., are based on the use of a richer approximation space regarding the number of parameters chosen in the sample. Clearly, the whole strategy of this approach relies on the fact that the solutions of the parametrized problems do not vary randomly with the parameters, but in fact vary in a smooth fashion.

\subsection{Global or pollution error versus local error}

In finite element analysis we may ask whether it is sufficient to just use a graded and reasonably refined mesh only around the point of interest. We will address this question somewhat in the context of the global error which has also been labeled the pollution error [6]. 
The global (or pollution) error can be defined as follows: Let $\mathscr{I}_{h} u$ denote the interpolant of the exact solution, then we can write

$e_{h}=\left(u-\mathscr{I}_{h} u\right)+\left(\mathscr{I}_{h} u-u_{h}\right)=e_{h, \mathrm{loc}}+e_{h, \mathrm{glob}}$

where $e_{h, \text { loc }}$ and $e_{h \text {,glob }}$ denote, respectively, the local error and the global error. Of course, in trivial 1D-problems the global error is zero if the finite element solution interpolates the exact solution at the nodes. However, in general, considering a structural analysis (elliptic) problem, the geometry, the loads, and boundary conditions have a global influence on the solution, and $e_{h \text {,glob }}$ cannot be expected to be zero. Indeed, we need to expect that this part of the error is large when there are stress singularities or high stress gradients in the solution that are not sufficiently resolved by the finite element solution.

To illustrate the global error numerically, we consider a bar of length $l$ with varying cross-section governed by the equation

$-\left(E A(x) u^{\prime}(x)\right)^{\prime}=f(x)$ for $0<x<l, \quad u(0)=u(l)=0$

This problem is similar to the problem studied by Babuška and Strouboulis [6], but we give further results. Here, we choose for the cross-sectional area

$A(x)=A_{1}+x \frac{A_{2}-A_{1}}{l} \quad A_{1}=t \cdot A_{2}$

to simulate a singular solution at $x=0$ if $t$ approaches zero, so that the solution is expected to be polluted. The exact solution of this model problem for different values of $t$ and chosen data is shown in Fig. 4. For our numerical studies we choose $t=0.01$, which, with the data in Fig. 4, yields the exact solution

$u(x)=-1 . \overline{01} x+0.219341 \ln (1+99 x)$

For the approximation we use eight linear finite elements on a uniform mesh with exact integration of the stiffness matrix for each element of varying cross-sectional area.

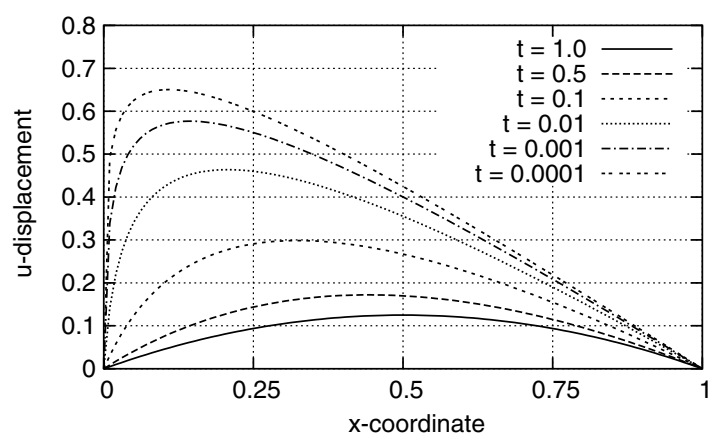

Fig. 4. Exact solutions for the bar with varying cross-section $A_{1}=t \cdot A_{2}\left(E=1, l=1, A_{2}=1, f=1\right)$.

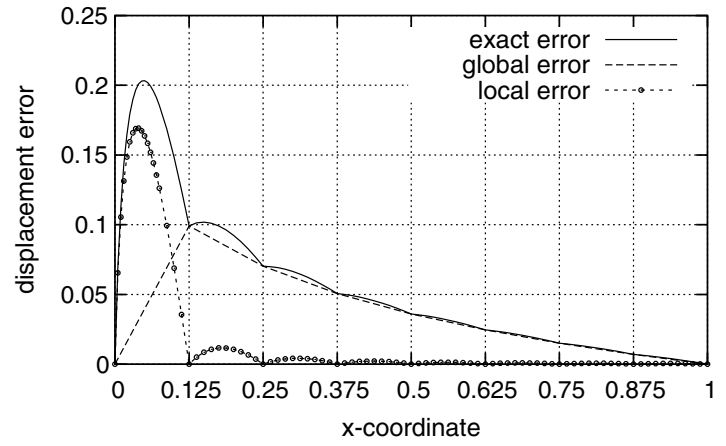

Fig. 5. Global and local errors for $t=0.01\left(E=1, l=1, A_{2}=1\right.$, $f=1$ ). Eight element solution.

The results in Fig. 5 show that only in the first element the local error is larger than the global error and elsewhere the local error becomes negligible; thus, the global error dominates in all other elements. Finally, in Fig. 6 we give only the global error with respect to the factor $t$. As expected, the global error decreases if the singularity in the exact solution decreases. Of course, for values of about $t<0.1$ the problem solved is not of much practical relevance.

To exemplify the global error in a $2 \mathrm{D}$ problem numerically we consider a plate in plane stress conditions (see Fig. 7). The mesh consists of bilinear 4-node elements. We compare the results for the given mesh with respect to a very fine finite element solution representing an almost exact reference solution. Fig. 8 shows the absolute values of the global error at different $y$-levels. Using these absolute values to evaluate percentage values, the maximum percent error in the $u_{x}$-displacement is $\sim 6 \%$ at the point $\mathrm{A}(x=4.5 / y=4.5)$ and $\sim 24 \%$ in the $u_{y}$-direction, and the percentage error decreases considerablysimilar as in the one-dimensional problem considered in Fig. 5-with increasing distance from the fixed corner

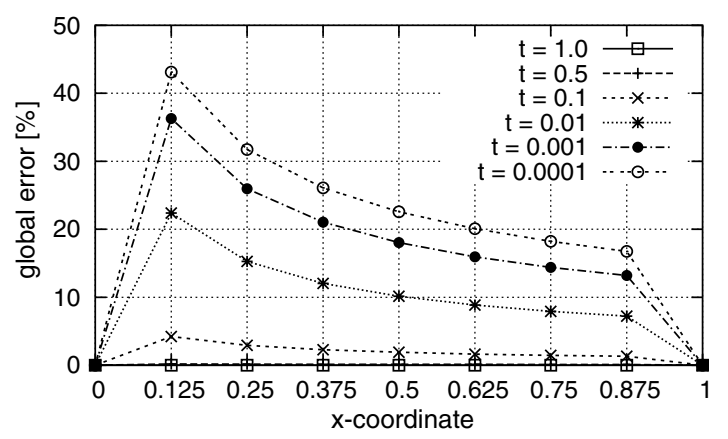

Fig. 6. Global error with varying cross-section $A_{1}=t \cdot A_{2}$ $\left(E=1, l=1, A_{2}=1, f=1\right)$. Eight element solution. 


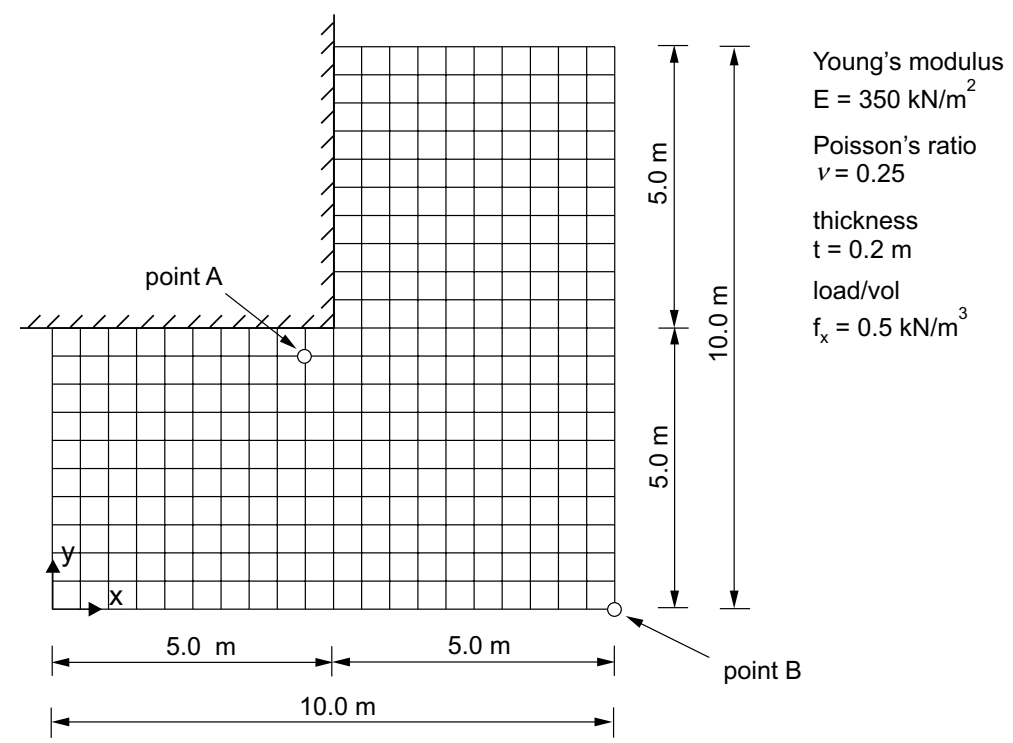

Fig. 7. Plate in plane stress condition to study the global error.
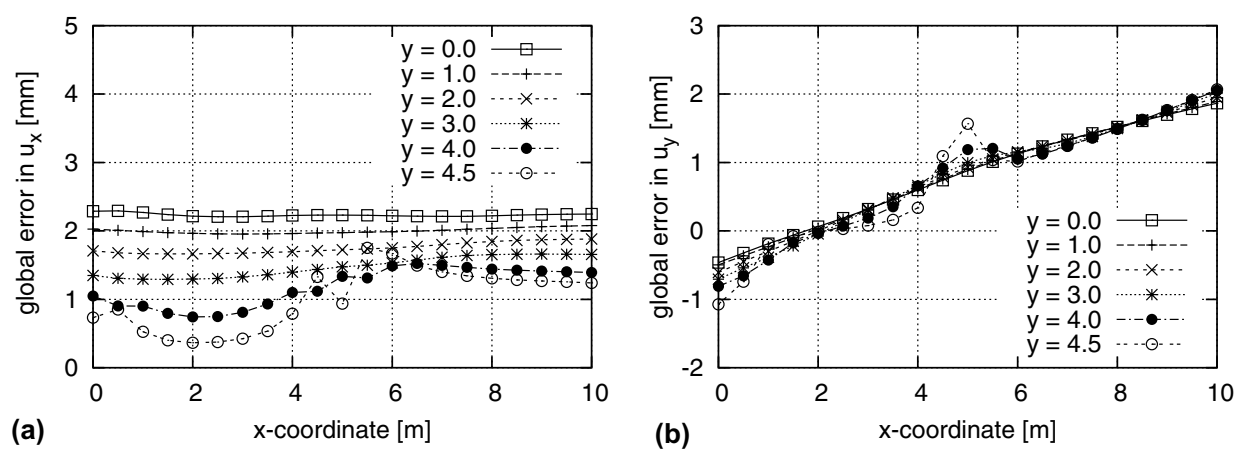

Fig. 8. Global error for the plate in plane stress condition: (a) error in $u_{x}$-displacement, (b) error in $u_{y}$-displacement.

towards the point B. At the point B the percentage error is $\sim 2 \%$ in both displacements, $u_{x}$ and $u_{y}$. Also, the global error would be smaller if the corner were not sharp as in a good practical design. For further details on this part of the error in 2D analysis see Ref. [6].

In practice, frequently the errors can successfully be controlled by refining the mesh in the neighborhood of the high stress gradients. Hence, to obtain an accurate approximation to the quantity of interest, we need to refine the mesh mostly around the domain of interest and at singularities. Indeed, in practice, the areas of high stress gradients are usually also the areas of interest and hence, natural mesh refinements in those areas frequently control the global error. By employing goal-oriented error estimation procedures the proper balance between the local error and the global error is always maintained and the mesh is refined to be most beneficial to the accuracy of the quantity of interest. Of course, this approach has the disadvantage that the mesh refinement is specific to only the one chosen goal quantity, whereas, in practice, the stresses over the complete analysis domain are frequently required.

\subsection{Concluding remarks}

Goal-oriented error estimation techniques focus on establishing accurate and computationally inexpensive error estimates for a quantity of interest. We conclude that any error estimator for the energy norm may be applied to goal-oriented error estimation procedures if (69) is used to bound the error and the experiences regarding these error estimators are applicable (see Section 4). 
Considering the dual-weighted residual method, the error estimate may over- or underestimate the actual error if the approximate error representations (86) or (87) are used. Error bounds as in (70) and (85) lead generally to an overestimation of the true error, since the cancellation effect of the error is neglected [60]. Moreover, the upper bound (85) is, strictly, not guaranteed if the unknown dual solution is approximated. However, in practice, the main issue of this error bound is the overestimation rather than providing a not guaranteed upper bound estimate.

When considering complex problems of practical interest, we can expect that the error bounds in (75) may lead to a rather large distance between the lower and upper bounds. In this regard, some results for 3D problems were recently published [61] but additional experiences need be obtained.

\section{Nonlinear problems}

In solving nonlinear problems by a Galerkin finite element method, we basically solve a series of linear problems in using an iteration scheme such as the Newton-Raphson method. Any of the error estimators derived in Sections 4 and 5 may therefore also - in principle - be employed to estimate the error in nonlinear problems. In the following we explain the basic ideas for a simple model problem and emphasize goal-oriented error estimates.

As a nonlinear model problem we choose a modification of the earlier model considered (see Section 3), namely the diffusion-reaction equation 4

$-\Delta u-u^{3}=f$ on $\Omega ; \quad u=0$ on $\Gamma$

The standard variational form is to find $u \in V$ such that $a(u ; \psi)=l(\psi) \quad \forall \psi \in V$

where $u$ is the exact (weak) solution, $a(. ;$.$) is a semi-lin-$ ear form that is linear only in the second argument, $l($.$) is$ a linear functional and $V$ is the appropriate Sobolev space. The semi-linear form in our model case is defined by

$a(u ; \psi)=(\nabla u, \nabla \psi)-\left(u^{3}, \psi\right)$

The corresponding finite element approximation uses finite dimensional subspaces $V_{h} \subset V$ to determine $u_{h} \in V_{h}$ such that

$a\left(u_{h} ; \psi_{h}\right)=l\left(\psi_{h}\right) \quad \forall \psi_{h} \in V_{h}$

\footnotetext{
${ }^{4}$ We employ this problem since there are no difficulties in proving the uniqueness and stability of the solution (see Ref. [62]), hence we can straightforwardly focus on the error estimate.
}

assuming that $u_{h}$ is the exact solution of (110). The error $e_{h}=u-u_{h}$ of the finite element approximation satisfies the error representation

$\mathscr{R}_{h}(\psi)=l(\psi)-a\left(u_{h} ; \psi\right) \quad \forall \psi \in V$

from which we obtain the Galerkin orthogonality

$\mathscr{R}_{h}\left(\psi_{h}\right)=l\left(\psi_{h}\right)-a\left(u_{h} ; \psi_{h}\right)=0 \quad \forall \psi_{h} \in V_{h}$

In order to linearize the error representation (111) with respect to $e_{h}$, we consider

$$
\begin{aligned}
\mathscr{R}_{h}(\psi) & =a(u ; \psi)-a\left(u_{h} ; \psi\right) \\
& =(\nabla u, \nabla \psi)-\left(u^{3}, \psi\right)-\left(\nabla u_{h}, \nabla \psi\right)+\left(u_{h}^{3}, \psi\right) \\
& =\left(\nabla e_{h}, \nabla \psi\right)-\left(u^{3}-u_{h}^{3}, \psi\right) \\
& =\left(\nabla e_{h}, \nabla \psi\right)-\left(e_{h}^{3}+3 u_{h}^{2} e_{h}+3 u_{h} e_{h}^{2}, \psi\right)
\end{aligned}
$$

and provided that the finite element solution $u_{h}$ is sufficiently close to the exact solution $u$, we obtain

$$
\mathscr{R}_{h}(\psi) \approx\left(\nabla e_{h}, \nabla \psi\right)-\left(3 u_{h}^{2} e_{h}, \psi\right)
$$

This error representation corresponds to the assumption that the error $e_{h}=u-u_{h}$ can be estimated by the solution of the linearized problem utilizing the displacement $w$ as an approximation to $u$ that satisfies

$a^{\prime}\left(u_{h} ; w-u_{h}, \psi\right)=\sum_{K \in \mathscr{T}_{h}}\left\{\int_{K} R \psi \mathrm{d} \Omega+\int_{\partial K} J \psi \mathrm{d} s\right\} \forall \psi \in V$

to which we can apply any of the error estimators presented in Section 4. In (118) we use the Gateaux-derivative of the semi-linear form with respect to the first argument defined by

$$
\begin{aligned}
a^{\prime}(u ; \varphi, \psi) & =\left[\frac{\mathrm{d}}{\mathrm{d} \varepsilon} a(u+\varepsilon \varphi ; \psi)\right]_{\varepsilon=0} \\
& =(\nabla \varphi, \nabla \psi)-\left(3 u^{2} \varphi, \psi\right)
\end{aligned}
$$

which is, clearly, the usual tangent form used in standard Newton iteration schemes [1].

To estimate the error in an individual (not necessarily linear) quantity of interest we distinguish between the error in the quantity

$Q(u)-Q\left(u_{h}\right)=a(z ; u)-a\left(z_{h} ; u_{h}\right)$

and the quantity of the error

$Q\left(e_{h}\right)=a\left(z ; e_{h}\right)=a(z ; u)-a\left(z ; u_{h}\right)$

But the solution $z$ in (120) and (121) cannot be interpreted as an influence function in the original meaning of linear analyses. In linear analysis, of course, (120) and (121) express the same relationships and hence it is prudent to linearize in order to estimate the error in the quantity of interest.

We therefore consider for a specified $u$ the dual problem: calculate $z \in V$ such that

$a^{\prime}(u ; z, \psi)=Q^{\prime}(u ; \psi) \quad \forall \psi \in V$ 
in which the functional is assumed to be Gateaux-differentiable, that is

$Q^{\prime}(u ; \psi)=\left[\frac{\mathrm{d}}{\mathrm{d} \varepsilon} Q(u+\varepsilon \psi)\right]_{\varepsilon=0}$

We then may seek the error in the goal quantity, with $\psi=w-u_{h}$,

$a^{\prime}\left(u_{h} ; z, w-u_{h}\right)=Q^{\prime}\left(u_{h} ; w-u_{h}\right)$

where $Q^{\prime}\left(u_{h} ; w-u_{h}\right)$ is a linear approximation of the true error in the quantity of interest at the linearization point. Invoking the standard Galerkin orthogonality yields

$$
\begin{aligned}
Q^{\prime}\left(u_{h} ; w-u_{h}\right)= & a^{\prime}\left(u_{h} ; w-u_{h}, z-z_{h}\right) \\
= & \sum_{K \in \mathscr{T}_{h}}\left\{\int_{K} R\left(z-z_{h}\right) \mathrm{d} \Omega\right. \\
& \left.+\int_{\partial K} J\left(z-z_{h}\right) \mathrm{d} s\right\}
\end{aligned}
$$

which can be employed in the dual-weighted residual method or, as well, in the Green's function decomposition method in case of point quantities. Clearly, employing the Cauchy-Schwarz inequality gives the error bound

$$
\left|Q^{\prime}\left(u_{h} ; w-u_{h}\right)\right| \leqslant\left\|z-z_{h}\right\|_{E}\left\|w-u_{h}\right\|_{E}
$$

It should be emphasized that as in the linear case we simply solve an additional load case at the linearization point in order to find an estimate for the error in a quantity of interest.

It is interesting to note that the linearized error representation (124) corresponds to a linearization of (121) with respect to $e_{h}$ if we proceed as in (113)-(117) for $\psi=z$ and assume that, as in the linear case, the semi-linear form $a(. ;$,$) is symmetric.$

A slightly different technique to estimate the error in a (nonlinear) target functional was proposed in $[33,34,63]$. This approach is based on the secant form

$a_{T}\left(u, u_{h} ; \varphi, \psi\right)=\int_{0}^{1} a^{\prime}\left(u_{h}+s e_{h} ; \varphi, \psi\right) \mathrm{d} s$

which is still bilinear in $\varphi$ and $\psi$. Using the secant form, the error $e_{h}=u-u_{h}$ is the solution of the variational problem

$a_{\mathrm{T}}\left(u, u_{h} ; e_{h}, \psi\right)=l(\psi)-a\left(u_{h} ; \psi\right)=\mathscr{R}_{h}(\psi) \quad \forall \psi \in V$

Here the exact solution $u$ on the left-hand side in (129) is unknown. One approach is to linearize about the finite element approximation, and then of course we obtain $a^{\prime}\left(u_{h} ; w-u_{h}, \psi\right)$ as used above.

To estimate the error in a functional of interest, we similarly define the secant form of the functional
$Q_{T}\left(u, u_{h} ; \psi\right)=\int_{0}^{1} Q^{\prime}\left(u_{h}+s e_{h} ; \psi\right) \mathrm{d} s$

where $Q_{T}$ is a linear functional in $\psi$. Here again $u$ is unknown and the straight linearization about $u_{h}$ of $Q_{T}\left(u, u_{h} ; \psi\right)$ results of course in obtaining $Q^{\prime}\left(u_{h} ; \psi\right)$. However, it is possible to use enhanced solutions instead of $u_{h}[4,63]$ and then a (somewhat) better approximation to the error may be obtained.

For estimating the error in inelastic analysis, notably elastoplasticity, several techniques have been presented, see for example [64-68] and the references therein. In general, in addition to the spatial approximation - the topic of this paper-also the errors resulting in the integration of the stresses and the evaluation of the constitutive relations need be considered. The estimation of the complete solution errors in general plasticity solutions is therefore much more difficult, and true error bounds are not yet available. However, the techniques reviewed above can directly be employed, as part of the procedures to estimate the complete error in inelastic analyses.

\section{Time-dependent problems}

The solution of time-dependent problems is of special interest in many engineering applications. From a numerical point of view, in addition to the usual approximation in space, also a time discretization scheme has to be applied. Our model transient problem is obtained from the original model problem in Section 3, and is the hyperbolic wave equation

$\ddot{u}(\mathbf{x}, t)-\beta \Delta u(\mathbf{x}, t)=\tilde{f}(\mathbf{x}, t)$ in $\Omega_{T}=\Omega \times I ; \quad I=(O, T)$

with $\beta$ a physical constant and the following initial and boundary conditions

$$
u(\mathbf{x}, 0)=u_{0} ; \quad \dot{u}(\mathbf{x}, 0)=\dot{u}_{0} \text { on } \Omega ; \quad u(\mathbf{x}, t)=0 \text { on } \Gamma
$$

These equations govern the dynamic motion of a prestressed membrane with $\beta=N / \rho, \tilde{f}=f / \rho$ where $N$ denotes the prestressing force, $\rho$ the mass density and $f$ is the load per unit area.

The commonly used finite element solution approach to solve hyperbolic differential equations is to approximate the problem with finite elements in space and to apply a finite difference scheme in time. The variational formulation of (131) and (132) is:

Find a function $u(\mathbf{x}, t)$ that is for all $t \in(O, T)$ in the trial space $V=H_{0}^{1}(\Omega)$ such that

$(\ddot{u}, v)+a(u, v)=l(v) \quad \forall v \in V$ 
subject to the weak initial conditions

$(u(\mathbf{x}, 0), v)=\left(u_{0}, v\right) \quad(\dot{u}(\mathbf{x}, 0), v)=\left(\dot{u}_{0}, v\right) \quad \forall v \in V$

The bilinear form and the right-hand side are defined as usual by

$a(u, v)=\int_{\Omega} \beta \nabla u \cdot \nabla v \mathrm{~d} \Omega \quad l(v)=\int_{\Omega} \tilde{f} v \mathrm{~d} \Omega$

Of course, the solution of (131) and (132) can also be obtained using a space and time variational formulation $[4,48,69,70]$, but such approach is hardly effective in engineering practice.

To obtain the finite element solution of (133) we employ the standard Galerkin method with the trial and test space $V_{h} \subset V$. The finite element solution lies in that space but is now time-dependent and denoted as $u_{h}(\mathbf{x}, t)$. The semi-discrete problem consists of seeking $u_{h}(\mathbf{x}, t)$ for all $t \in(O, T)$ in $V_{h}$ such that

$$
\begin{aligned}
& \left(\ddot{u}_{h}, v_{h}\right)+a\left(u_{h}, v_{h}\right)=l\left(v_{h}\right) \\
& \begin{aligned}
\left(u_{h}(\mathbf{x}, 0), v_{h}\right) & =\left(u_{0}, v_{h}\right) \quad\left(\dot{u}_{h}(\mathbf{x}, 0), v_{h}\right) \\
& =\left(\dot{u}_{0}, v_{h}\right) \forall v_{h} \in V_{h}
\end{aligned}
\end{aligned}
$$

Eqs. (136) and (137) represent a system of ordinary differential equations in time which can be treated by standard finite difference schemes.

As an example, consider the Newmark method, which is used widely [1]. Here, the total time interval $(O, T)$ is subdivided into $n$ equal time intervals $\Delta t$ and the solution is calculated at discrete times $\Delta t, 2 \Delta t, \ldots, t$, $t+\Delta t, \ldots, T$. The following assumptions to calculate the velocities and displacements are used

$$
\begin{aligned}
& { }^{t+\Delta t} \dot{\mathbf{u}}={ }^{t} \dot{\mathbf{u}}+\left[(1-\delta)^{t} \ddot{\mathbf{u}}+\delta^{t+\Delta t} \ddot{\mathbf{u}}\right] \Delta t \\
& { }^{t+\Delta t} \mathbf{u}={ }^{t} \mathbf{u}+{ }^{t} \dot{\mathbf{u}} \Delta t+\left[(1 / 2-\alpha)^{t} \ddot{\mathbf{u}}+\alpha^{t+\Delta t} \ddot{\mathbf{u}}\right] \Delta t^{2}
\end{aligned}
$$

with Newmark parameters $\alpha$ and $\delta$. Inserting (138) and (139) into (136) leads to a fully discretized form in space and time giving the solution at the time $t+\Delta t$.

Now the actual error $e_{h}=u-u_{h}$ involves both discretization errors in space and discretization errors in time [71]. In this paper we are only concerned with the spatial discretization error at a given time, and this error can be estimated with the error procedures discussed above. However, the spatial error is only a part of the total error and hence this estimation is only of limited value. The overall errors to represent the required frequencies and integrate accurately in time are not assessed by the procedures we discussed and represent major sources of errors in the solution [1].

Finally, we should mention that the ideas described above can directly be used in the solution of certain parabolic problems such as the model heat-conduction equation

$$
\begin{gathered}
\dot{u}(\mathbf{x}, t)-\beta^{\star} \Delta u(\mathbf{x}, t)=f(\mathbf{x}, t) \\
\quad \text { in } \Omega_{T}=\Omega \times I ; \quad I=(O, T)
\end{gathered}
$$

where $\beta^{\star}$ is a physical constant.

\section{Mixed formulations}

To be somewhat complete in our presentation, we briefly discuss how error estimation procedures might be applied in mixed formulations. Usually, we refer to a mixed formulation if the problem is based on a twofield formulation with respect to the solution. For our simple model problem (see Section 3), assuming $u=0$ on $\Gamma$, we might seek the pair $\mathbf{w}=\{u, \sigma\}$ that satisfies the system of equations

$$
\begin{aligned}
& \nabla u-\boldsymbol{\sigma}=\mathbf{0} \\
& -\operatorname{div} \boldsymbol{\sigma}=f
\end{aligned}
$$

An equivalent formulation of the boundary value problem is the mixed variational formulation seeking the pair $\mathbf{w}=\{u, \boldsymbol{\sigma}\} \in V \times W$ such that

$(\boldsymbol{\sigma}, \tau)+(u, \operatorname{div} \tau)=0$

$(\operatorname{div} \boldsymbol{\sigma}, v)=l(v)$

for all $\varphi=\{v, \tau\} \in V \times W$ and $l(v)=-(f, v)$. Here the trial and test spaces are defined by $V=L^{2}(\Omega)$ and $W=H^{\operatorname{div}}=\left\{\tau \in L^{2}(\Omega) ; \quad \operatorname{div} \tau \in L^{2}(\Omega)\right\} \quad$ where $W$ is equipped with the norm $\|\tau\|_{H^{\mathrm{div}}}^{2}=\|\tau\|_{L^{2}(\Omega)}^{2}+\|\operatorname{div} \tau\|_{L^{2}(\Omega)}^{2}$.

We assume that the solution of the mixed variational problem exists and is unique, i.e., the stability conditions

$(\tau, \tau) \geqslant \alpha\|\tau\|_{W}^{2} \quad \tau \in N$

$\inf _{v \in V} \sup _{\tau \in W} \frac{(\operatorname{div} \tau, v)}{\|\tau\|_{W}\|v\|_{V}} \geqslant \beta$

are satisfied with positive constants $\alpha, \beta$ and $N=\{\tau \in W ;(\operatorname{div} \tau, v)=0 \forall v \in V\}$. Of course, the condition (146) is known as the continuous inf-sup condition (see e.g. Refs. [72-74]).

The discrete mixed formulation seeks a pair $\mathbf{w}_{h}=\left\{u_{h}, \boldsymbol{\sigma}_{h}\right\} \in V_{h} \times W_{h}$ such that

$\left(\boldsymbol{\sigma}_{h}, \tau_{h}\right)+\left(u_{h}, \operatorname{div} \tau_{h}\right)=0$

$\left(\operatorname{div} \sigma_{h}, v_{h}\right)=l\left(v_{h}\right)$

for all $\boldsymbol{\varphi}_{h}=\left\{v_{h}, \tau_{h}\right\} \in V_{h} \times W_{h}$ with $V_{h} \subset V$ and $W_{h} \subset W$. The crucial point for the solvability, stability and optimality of the finite element approximation is that the finite element spaces satisfy the discrete infsup condition [72-74]

$\inf _{v_{h} \in V_{h}} \sup _{\tau_{h} \in W_{h}} \frac{\left(\operatorname{div} \tau_{h}, v_{h}\right)}{\left\|\tau_{h}\right\|_{W_{h}}\left\|v_{h}\right\|_{V_{h}}} \geqslant C>0$ 
Then the solution is optimal in the sense

$$
\begin{aligned}
\| \boldsymbol{\sigma} & -\boldsymbol{\sigma}_{h}\left\|_{W}+\right\| u-u_{h} \|_{V} \\
& \leqslant c\left\{\inf _{\tau_{h} \in W_{h}}\left\|\boldsymbol{\sigma}-\tau_{h}\right\|_{W}+\inf _{v_{h} \in V_{h}}\left\|u-v_{h}\right\|_{V}\right\}
\end{aligned}
$$

Clearly, it is possible to formulate the mixed variational problem as follows

$$
\begin{aligned}
A(\mathbf{w}, \boldsymbol{\varphi}) & =(\boldsymbol{\sigma}, \tau)+(u, \operatorname{div} \tau)+(\operatorname{div} \boldsymbol{\sigma}, v) \\
& =l(v) \quad \forall \boldsymbol{\varphi} \in V \times W
\end{aligned}
$$

with the symmetric bilinear form $A(.,$.$) . Then for the$ mixed finite element approximation we have

$A\left(\mathbf{w}_{h}, \boldsymbol{\varphi}_{h}\right)=l\left(v_{h}\right)$

which directly leads to the Galerkin orthogonality for the error $\mathbf{e}_{h}=\mathbf{w}-\mathbf{w}_{h}$

$$
\begin{aligned}
A\left(\mathbf{e}_{h}, \boldsymbol{\varphi}_{h}\right) & =\left(\boldsymbol{\sigma}-\boldsymbol{\sigma}_{h}, \tau_{h}\right)+\left(u-u_{h}, \operatorname{div} \tau_{h}\right)+\left(\operatorname{div}\left(\boldsymbol{\sigma}-\boldsymbol{\sigma}_{h}\right), v_{h}\right) \\
& =0 \quad \forall \boldsymbol{\varphi}_{h} \in V_{h} \times W_{h}
\end{aligned}
$$

To derive an a posteriori error estimator for the mixed problem we proceed as in Section 4.1. Thus, we obtain the following error representation

$$
A\left(\mathbf{e}_{h}, \boldsymbol{\varphi}\right)=l(v)-A\left(\mathbf{w}_{h}, \boldsymbol{\varphi}\right)=\mathscr{R}_{h}(\boldsymbol{\varphi})
$$

to hold true for each pair $\varphi=\{v, \tau\} \in V \times W$. If the domain integral is split into its contributions from each element, (154) can be rewritten as

$$
\begin{aligned}
A\left(\mathbf{e}_{h}, \boldsymbol{\varphi}\right)= & \sum_{K \in \mathscr{T}_{h}}\left\{-\int_{K} f v \mathrm{~d} \Omega-\int_{K} \boldsymbol{\sigma}_{h} \cdot \tau \mathrm{d} \Omega\right. \\
& \left.-\int_{K} u_{h} \operatorname{div} \tau \mathrm{d} \Omega-\int_{K} \operatorname{div} \boldsymbol{\sigma}_{h} v \mathrm{~d} \Omega\right\}
\end{aligned}
$$

Applying integration by parts to the third integral and assuming the finite element solution $u_{h}$ is sufficiently smooth on $K$ yields

$$
\int_{K} u_{h} \operatorname{div} \tau \mathrm{d} \Omega=\int_{\partial K} u_{h} \mathbf{n} \cdot \tau \mathrm{d} s-\int_{K} \nabla u_{h} \cdot \tau \mathrm{d} \Omega
$$

The boundary term does not vanish after summing over all elements because the finite element solution $u_{h}$ is only in $L^{2}(\Omega)$. Yet the expression $\mathbf{n} \cdot \tau$ is continuous at the element boundaries since $\tau \in H^{\mathrm{div}}(\Omega)$. Rearranging terms yields

$$
\begin{aligned}
A\left(\mathbf{e}_{h}, \boldsymbol{\varphi}\right)= & \sum_{K \in \mathscr{T}_{h}}\left\{\int_{K} R v \mathrm{~d} \Omega+\int_{K} \zeta \cdot \tau \mathrm{d} \Omega\right\} \\
& +\sum_{\gamma \in \partial \mathscr{T}_{h}} \int_{\gamma} J \mathbf{n} \cdot \tau \mathrm{d} s \quad \forall \boldsymbol{\varphi} \in V \times W
\end{aligned}
$$

where the element residuals are now defined by

$R=-\operatorname{div} \sigma_{h}-f$ in $\mathrm{K}$

$\zeta=-\sigma_{h}+\nabla u_{h} \quad$ in $\mathrm{K}$ and $J$ is the jump of the finite element solution $u_{h}$ across the element edge $\gamma$ of elements $K$ and $K^{\prime}$

$J= \begin{cases}\left(u_{h}-u_{h}^{\prime}\right) & \text { if } \gamma \not \Gamma \Gamma \\ 0 & \text { if } \gamma \subset \Gamma\end{cases}$

Note that the normal vector $\mathbf{n}$ and the jumps change sign if the orientation of the edge is reversed.

For driving an adaptive mesh iteration scheme, the following local error indicators might now be used for this model problem

$\eta_{K}^{2}=h_{K}^{2}\|R\|_{L^{2}(K)}^{2}+\|\zeta\|_{L^{2}(K)}^{2}+h_{K}\|J\|_{L^{2}(\partial K)}^{2}$

where on interelement boundaries $\partial K \nsubseteq \Gamma$ the jump $J$ is multiplied by $1 / 2$ to distribute the error equally onto the two elements sharing the common edge.

Similar error indicators can be established for other mixed formulations (see e.g. Ref. [75]), but the difficulty in complex practical engineering analysis is to truly measure the error in appropriate norms and establish error bounds. Some discussion in this regard is given in Refs. $[50,76]$.

To obtain error estimates for the solution of a linear quantity of interest $Q(\mathbf{w})$, we use a slightly different procedure than in the standard approach. Assuming that the functional is well defined on the solution space $V \times W$, the corresponding dual problem is:

Find $\mathbf{z}=\{z, \pi\} \in V \times W$ such that

$$
\begin{aligned}
Q(\boldsymbol{\varphi}) & =(\boldsymbol{\pi}, \boldsymbol{\tau})+(z, \operatorname{div} \tau)+(\operatorname{div} \pi, v) \\
& =A(\mathbf{z}, \boldsymbol{\varphi}) \quad \forall \boldsymbol{\varphi} \in V \times W
\end{aligned}
$$

where $\mathbf{z}$ is the dual solution and $\nabla z-\pi=\mathbf{0}$. Taking $\boldsymbol{\varphi}=\mathbf{e}_{h}$ and utilizing the Galerkin orthogonality (153) yields the error identity

$$
\begin{aligned}
Q\left(\mathbf{e}_{h}\right)= & \left(\left(\boldsymbol{\pi}-\boldsymbol{\pi}_{h}\right),\left(\boldsymbol{\sigma}-\boldsymbol{\sigma}_{h}\right)\right)+\left(z-z_{h}, \operatorname{div}\left(\boldsymbol{\sigma}-\boldsymbol{\sigma}_{h}\right)\right) \\
& +\left(\operatorname{div}\left(\boldsymbol{\pi}-\boldsymbol{\pi}_{h}\right), u-u_{h}\right) \\
= & A\left(\mathbf{z}-\mathbf{z}_{h}, \mathbf{w}-\mathbf{w}_{h}\right)
\end{aligned}
$$

To find an estimate for this quantity, we could write

$$
\begin{aligned}
\left|Q\left(\mathbf{e}_{h}\right)\right| & =\left|A\left(\mathbf{z}-\mathbf{z}_{h}, \mathbf{w}-\mathbf{w}_{h}\right)\right| \\
& \leqslant\left\|\mathbf{z}-\mathbf{z}_{h}\right\|\left\|\mathbf{w}-\mathbf{w}_{h}\right\|
\end{aligned}
$$

and apply some error estimators for the norm $\|$.$\| . How-$ ever, note that we have $\|\varphi\| \neq \sqrt{A(\boldsymbol{\varphi}, \boldsymbol{\varphi})}$ since the bilinear form is not positive definite. Alternatively, we might follow the suggestions of Rannacher and Suttmeier in $[35,68]$, who propose a method that is directly related to the dual-weighted residual method described in Section 5.2.2.

\section{Numerical examples}

In this section, we give some example solutions to illustrate the use of error measures. Although a scalar 


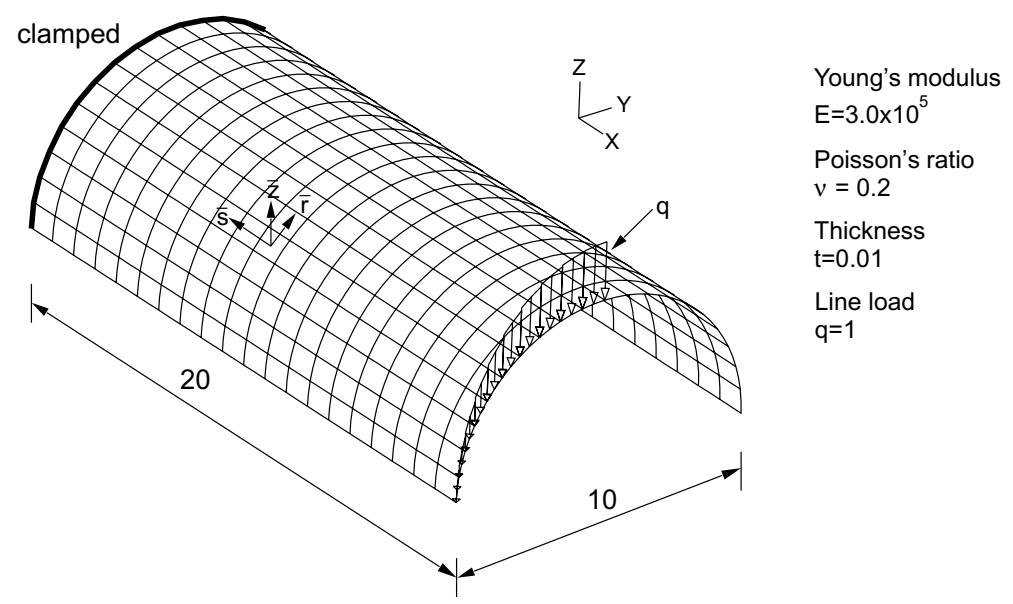

Fig. 9. Goal-oriented error measures for a shell structure: problem description.

model problem is considered in this paper, we consider in our numerical examples 2D linear elasticity and shell problems. We do not report upon actual practical engineering analyses but only give illustrative examples. Also, we are looking in some cases at very small errors, smaller than needed in practice, but do so because we want to study the convergence behavior of the procedures used.

\subsection{Analysis of a shell}

We consider a cylindrical shell that is partially clamped and loaded by a vertical line load as shown in Fig. 9. First, we study the results of a finite element computation on a reasonably fine mesh consisting of $20 \times 20$ MITC9 elements [51,52]. Fig. 10 shows that high stress concentrations are present in two regions near the clamped boundary (corresponding to the membrane stresses) and at the tip of the structure where the loading boundary conditions change (corresponding to the bending moment). Hence, using uniform MITC4 element meshes [52] and employing goal-oriented error estimates based on (90), we want to evaluate the following quantities of interest (see also Fig. 11):

$$
\begin{aligned}
& Q_{1}(\mathbf{U})=\left|\Omega_{1}\right|^{-1} \int_{\Omega_{1}} \sigma_{\overline{s s}}(\mathbf{U}) \mathrm{d} \Omega \\
& Q_{2}(\mathbf{U})=\left|\Omega_{2}\right|^{-1} \int_{\Omega_{2}} \sigma_{\overline{s s}}(\mathbf{U}) \mathrm{d} \Omega \\
& Q_{3}(\mathbf{U})=\left|\Omega_{3}\right|^{-1} \int_{\Omega_{3}} \bar{z} \sigma_{\overline{r r}}(\mathbf{U}) \mathrm{d} \Omega
\end{aligned}
$$

These quantities are evaluated using the local Cartesian coordinate system $(\bar{r}, \bar{s}, \bar{z})$ (see Fig. 9). The reference solution was obtained using a uniform mesh of $100 \times 100$ MITC9 elements (with 201,000 degrees of freedom) for the complete structure. For measuring the accuracy of our error estimate we use the effectivity index, which is the ratio between the estimated error using (90) and the calculated error using the reference solution defined in (89). As seen in Fig. 12 for every quantity of interest the estimated relative percentage error decreases quickly and the corresponding effectivity indices are close to 1.0.

Fig. 13 shows the predicted pointwise accuracy of the influence function for the quantity of interest defined in (165) when using the $20 \times 20$ mesh. We consider the absolute percentage value of the approximate error of the influence function normalized with the quantity of interest, $\left(\mathbf{Z}_{h}^{(M 9)}-\mathbf{Z}_{h}\right) / Q_{2}(\mathbf{U})$, and we define a tolerance on the absolute value, e.g. tol $=1.0 \%$. Then the grey regions indicate that the error in the quantity of interest is smaller than the tolerance if the load is applied there, while the white areas correspond to errors larger than the tolerance. For additional shell analyses using this approach of error estimation see Ref. [49].

\subsection{Analysis of a frame structure}

Fig. 14 shows the frame structure considered. We want to employ adaptive mesh refinement based on energy norm estimates and the goal-oriented techniques. For the analysis we use the 4-node displacement-based bilinear element. The special focus is on the integrated shear stress in the two sections A-A at level $y=4.0$, so that the quantity of interest is:

$Q\left(u_{h}\right)=\int_{0}^{2} \sigma_{x y}^{h} \mathrm{~d} x+\int_{14}^{16} \sigma_{x y}^{h} \mathrm{~d} x$

Local equilibrium asserts that the exact value is 144 which is equal to the volume above the cross-section times the load. Clearly, the finite element solution yields 

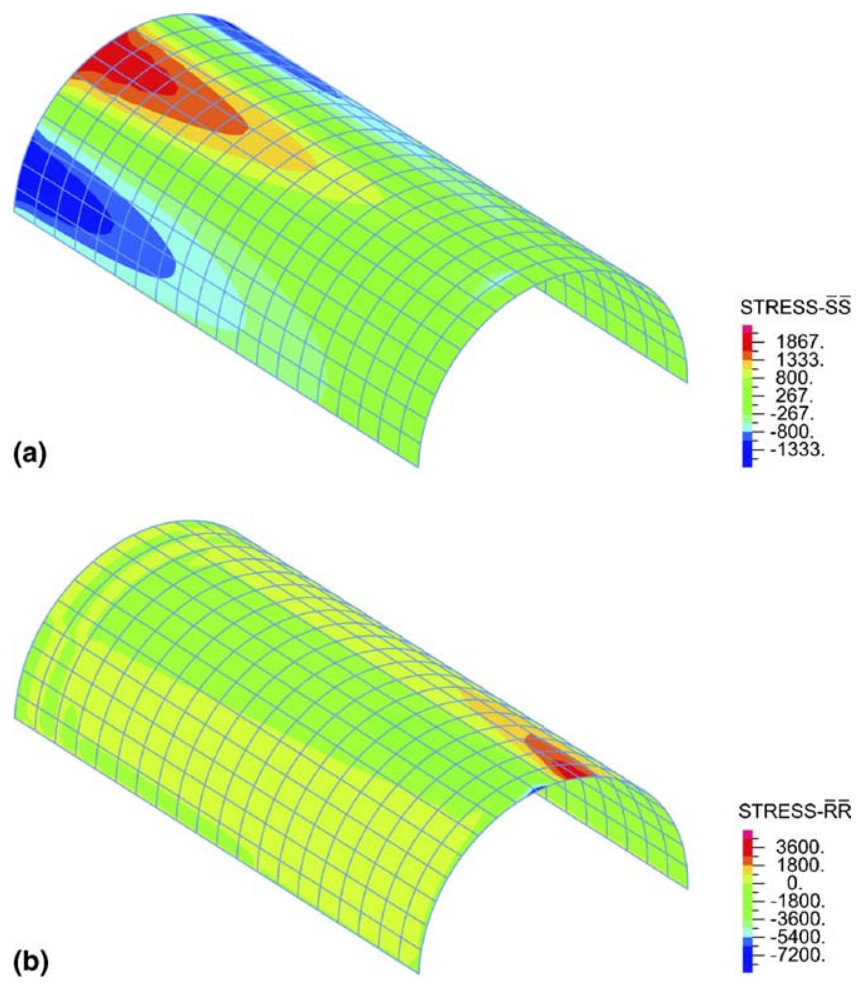

Fig. 10. Stresses of the shell structure under line load: (a) $\sigma_{\bar{s} \bar{s}}$-stress field and (b) $\sigma_{\overline{r \bar{r}}}$-stress field.

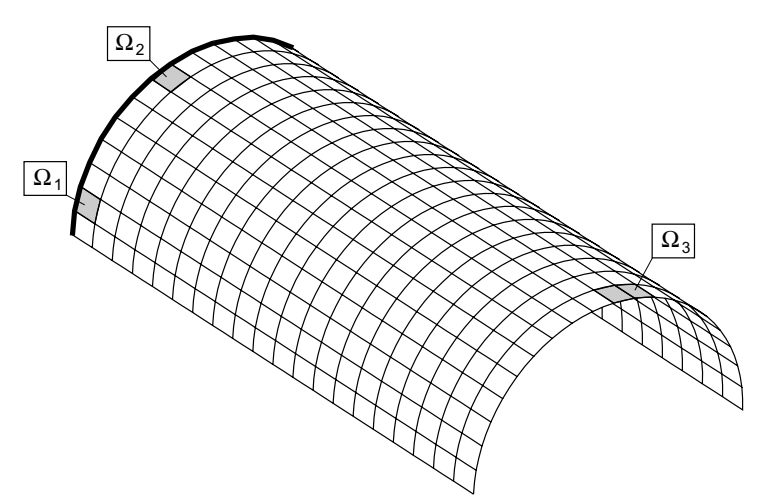

Fig. 11. Goal-oriented error measures for a shell structure: locations of three quantities of interest. $20 \times 20$ mesh shown.

only an approximation for the integrated stresses because the stresses in the element interiors and the fluxes at the boundaries are only approximations to the true values. Note that, of course, the finite element nodal forces in the cross-section $\mathrm{A}-\mathrm{A}$ are in exact equilibrium with the applied load [1].

For the solution we use two approaches: first, the refinement is based on the norms given in (28) and (29) and, second, the refinement is based on the goal-ori- ented error indicators given in (77)-(79). In both approaches we use the constants ${ }^{5}[13]$

$c_{1}=\frac{0.16}{\lambda+5 \mu} \quad c_{2}=\frac{1.44}{\lambda+5 \mu} \quad \lambda, \mu=$ Lamé constants

and the hanging node concept, in which the unknown displacements at a hanging node (a node not shared by all elements surrounding it) are eliminated by interpolation using the neighboring nodes. Also, in the approaches, the refinement criteria defined in (31) and (81) are used, respectively, and the $30 \%$ of the elements with $\xi_{K}>1$ (and corresponding to the largest values of $\left.\xi_{K}\right)$ are refined.

Fig. 15 compares the results obtained using these two solution approaches with the results obtained by simply using a uniform mesh refinement. In all these solutions, the quantity of interest is obtained by differentiation of the finite element displacement field to evaluate the stresses. Fig. 15 shows that the goal-oriented approach leads,

\footnotetext{
5 The numerator in the definition of the constant $c_{2}$ in (168) is changed compared to the value of 1.21 given in [13] since we are using quadrilaterals instead of triangles, for which the constants originally were derived.
} 

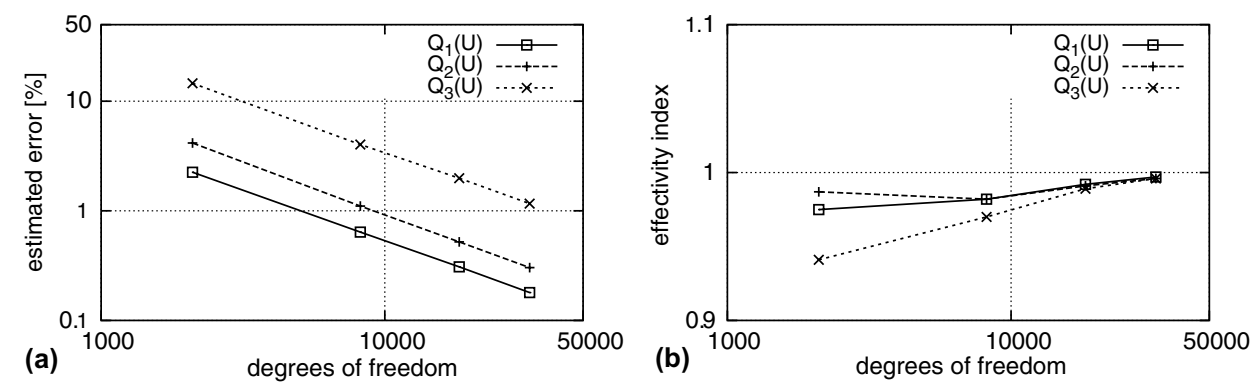

Fig. 12. Results of goal-oriented error estimation for the shell structure shown in Fig. 9: (a) estimated (absolute) relative errors in the quantities of interest and (b) corresponding effectivity indices. The numbers of degrees of freedom refer to the uniform MITC4 element meshes used for the complete shell.
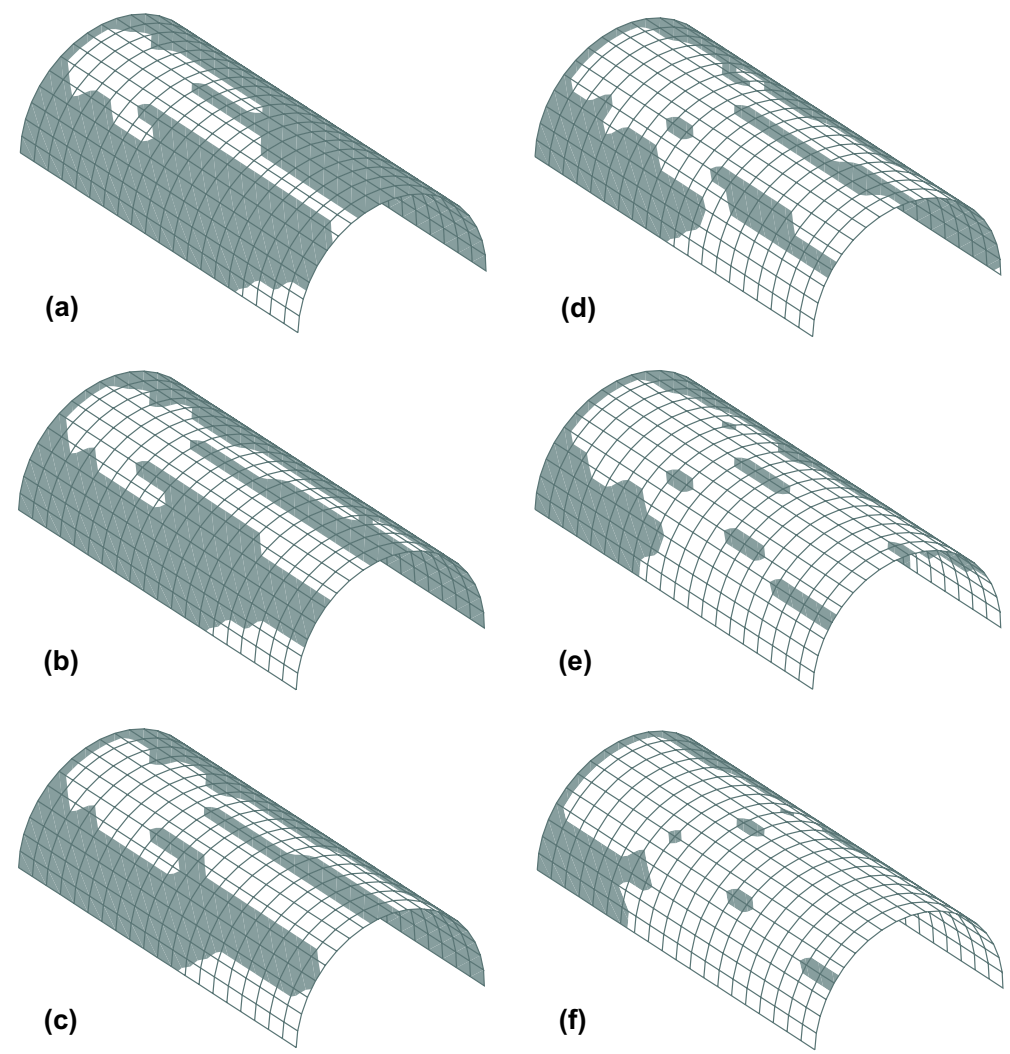

Fig. 13. Predicted absolute pointwise accuracy of the influence function normalized with the quantity of interest defined in (165): body loads in the $z$-direction in the grey region yield an error in the quantity of interest smaller than a tolerance of (a) tol $=1.0 \%$, (b) tol $=0.8 \%$, (c) tol $=0.6 \%$, (d) tol $=0.4 \%$, (e) tol $=0.2 \%$, (f) tol $=0.1 \%$.

as expected, to the smallest error in the quantity of interest. The initial and final meshes are shown in Fig. 16. The refinement based on (28) and (29) did not refine in the cross-section but at the singularities of the structure, whereas the density of the goal-oriented mesh obtained using (77)-(79) is the highest in the cross-section. Actually, for the range of degrees of freedom considered, there is only a slight improvement in the quantity of interest employing the energy norm for refinement of the mesh.

The stress error indicator in ADINA plotted in Fig. 16(a) is obtained by taking the difference between the maximum and minimum von Mises stress at the nodes, normalized to the maximum value [22]. These results 


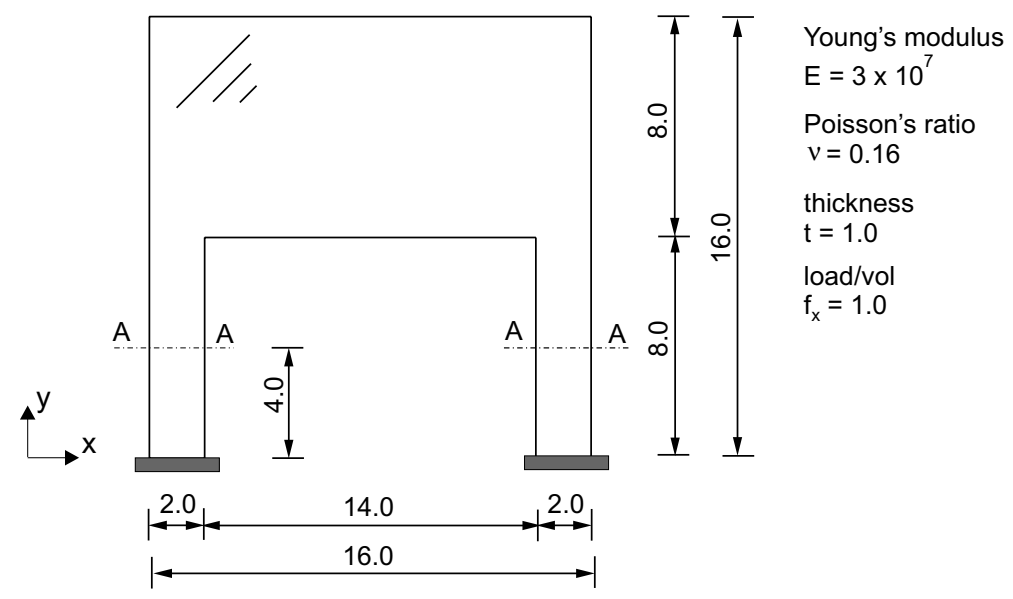

Fig. 14. Frame under horizontal body load to study the error in the integrated shear stresses in the two sections A-A.

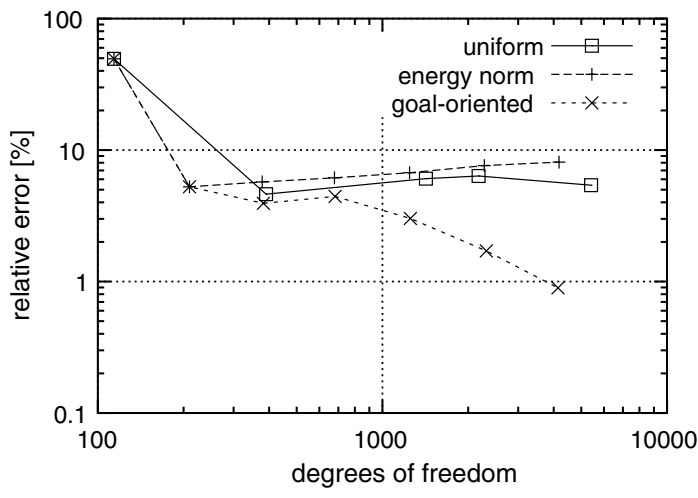

Fig. 15. Comparison of different adaptive refinements to approximate the integrated shear stresses in the two sections A-A. show that, as expected, the error indicator for the von Mises stress obtained with ADINA corresponds quite well with the energy norm refinement, so that, indeed, this error indicator would have provided a good guide for driving a mesh refinement "by-hand" without any usage of an error estimator for the energy norm.

Comparing the computational effort we need to keep in mind that for the goal-oriented approach two finite element solutions per mesh are necessary. However, the solution of the dual problem differs only in a modified load vector from the analysis of the primal problem; that is, the stiffness matrix remains the same.

\subsection{Analysis of a plate in plane stress}

Next, we study the example described in Fig. 17. The focus is on the stresses $\sigma_{x x}$ and $\sigma_{y y}$ at the point $\mathrm{A}$. The
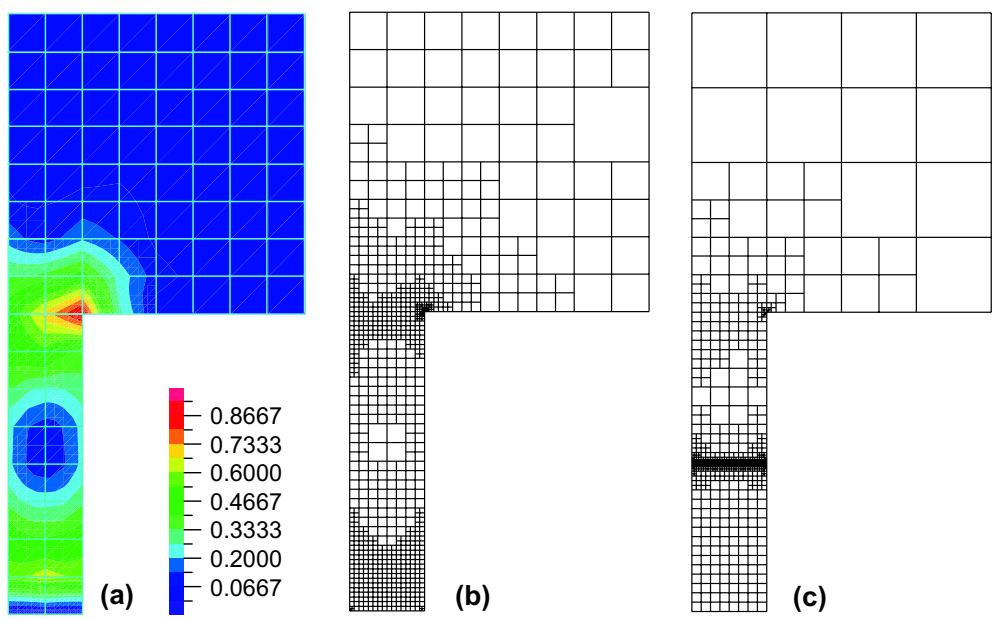

Fig. 16. Results obtained in the analysis of the frame in Fig. 15: (a) Initial mesh and error indicators obtained with ADINA, (b) final mesh obtained with the energy norm control and (c) final mesh obtained with the goal-oriented control. 


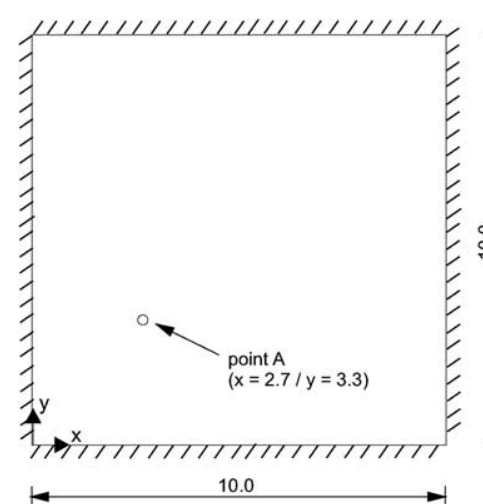

(a)

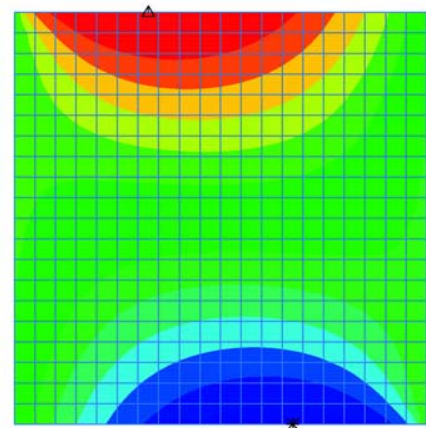

(c)
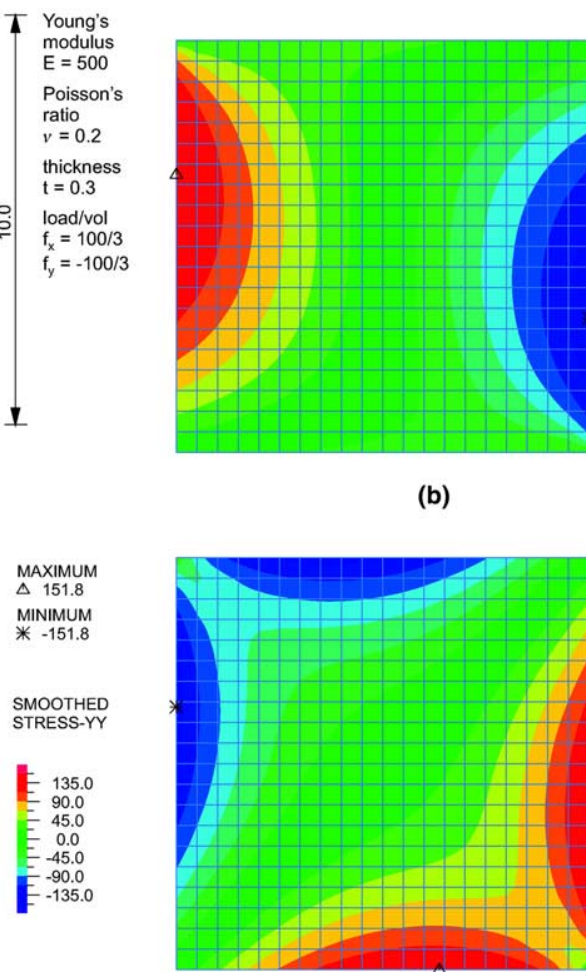

(d)

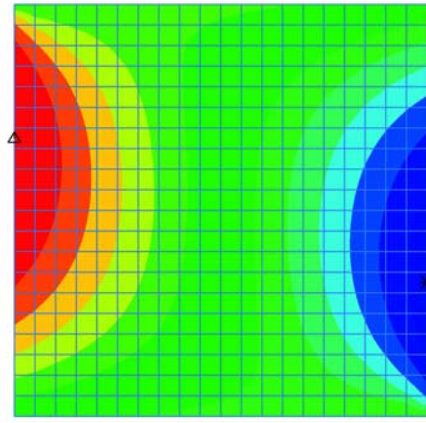

MAXIMUM MINIMUM * -151.8

SMOOTHED
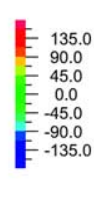

(b)

MAXIMUM
$\triangle 77.19$

MINIMUM

SMOOTHED

STRESS-XY

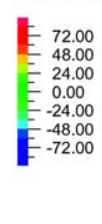

Fig. 17. Plate in plane stress condition to study the error in the stresses at the point A: (a) problem data, (b) $\sigma_{x x}$-stress field, (c) $\sigma_{y y^{-}}$ stress field and (d) $\sigma_{x y}$-stress field.

Table 1

Stresses at A employing the Green's function decomposition approach using uniform meshes

\begin{tabular}{lrllll}
\hline$h$ & DOF & $\sigma_{x x}$ & $\left|e_{\text {rel }}\right|[\%]$ & $\sigma_{y y}$ & $\left|e_{\text {rel }}\right|[\%]$ \\
\hline $1 / 5$ & 32 & 53.8018 & $2.931 \cdot 10^{-1}$ & -33.9038 & $1.055 \cdot 10^{0}$ \\
$1 / 10$ & 162 & 53.6705 & $4.884 \cdot 10^{-2}$ & -33.6288 & $2.349 \cdot 10^{-1}$ \\
$1 / 15$ & 392 & 53.6540 & $1.808 \cdot 10^{-2}$ & -33.5831 & $9.866 \cdot 10^{-2}$ \\
$1 / 20$ & 722 & 53.6492 & $9.134 \cdot 10^{-3}$ & -33.5681 & $5.395 \cdot 10^{-2}$ \\
$1 / 25$ & 1152 & 53.6473 & $5.592 \cdot 10^{-3}$ & -33.5614 & $3.398 \cdot 10^{-2}$ \\
$1 / 32$ & 1922 & 53.6460 & $3.169 \cdot 10^{-3}$ & -33.5569 & $2.057 \cdot 10^{-2}$ \\
$1 / 40$ & 3042 & 53.6454 & $2.051 \cdot 10^{-3}$ & -33.5544 & $1.311 \cdot 10^{-2}$ \\
$1 / 50$ & 4802 & 53.6450 & $1.305 \cdot 10^{-3}$ & -33.5528 & $8.346 \cdot 10^{-3}$ \\
Reference value & - & 53.6443 & - & -33.5500 & - \\
\hline
\end{tabular}

reference stress fields are obtained using a very fine mesh. In all analyses the 4-node displacement-based bilinear element is used.

First, we give some results obtained employing the Green's function decomposition method using uniform meshes. As a result of this uniform refinement shown in Table 1, the error in the stresses decreases reasonably fast, and indeed highly accurate results are achieved even on coarse meshes.
It is interesting to compare the results obtained with the Green's function decomposition method given in Table 1 with the results obtained using different mesh refinement techniques (see Fig. 18(a)). First, we calculate finite element approximations on uniformly refined meshes. Next, the refinement is steered using the global energy norm control based on the explicit error estimator in (28) and (29) with the constants given in (168), and in a third solution the refinement is obtained using the 

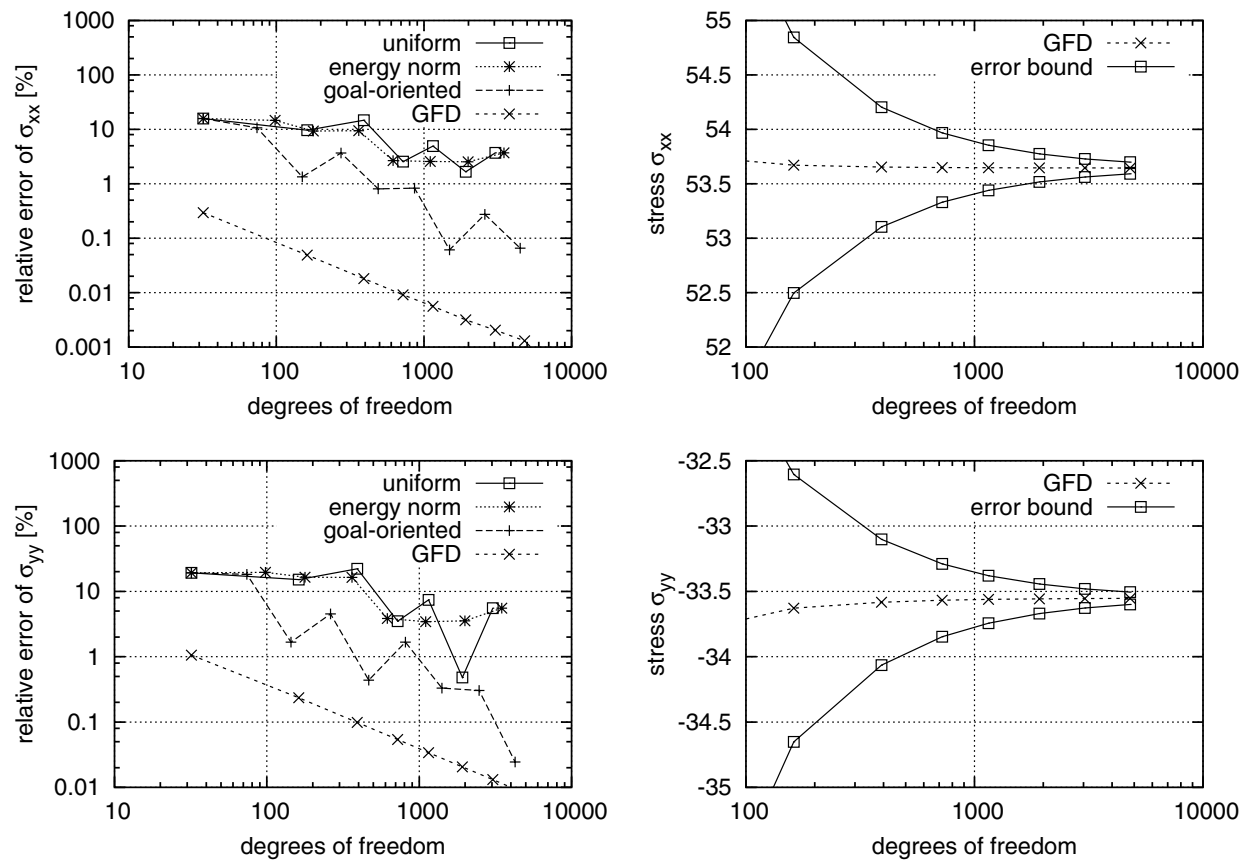

(a)

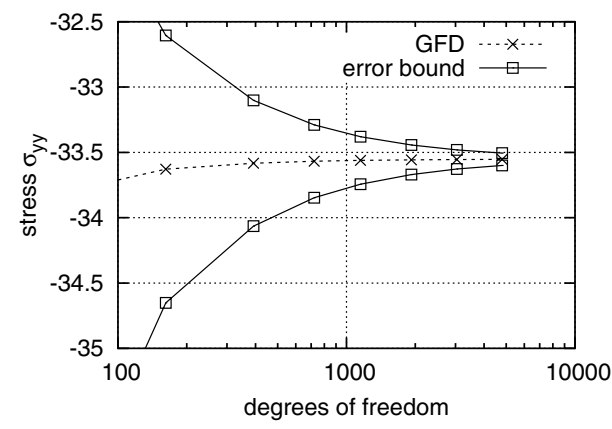

(b)

Fig. 18. Comparison of results obtained using different mesh refinement techniques for the stresses at point A: (a) relative error in stresses, (b) related error bounds (GFD are the Green's function decomposition method results in Table 1).

energy-norm-based goal-oriented strategy based on (77) using also the constants in (168). For the energy norm control, the refinement criterion in (31) is used, while for the energy-norm-based goal-oriented strategy the refinement criterion given in (81) is employed. In both approaches $25 \%$ of the elements with $\xi_{K}>1$ (and corresponding to the largest values of $\xi_{K}$ ) are refined.

In the goal-oriented error estimate, to solve the dual problem in (64) and the corresponding nodal forces in (65), we consider integrated stresses over a small circular domain and take numerically the limit value as the radius of the domain tends to zero. For evaluating the stress quantity on a given mesh, we simply differentiate the finite element displacement field, as usual in finite element analysis.

As can be seen in Fig. 18, the stresses obtained with the uniformly refined meshes, the global energy norm control, and the goal-oriented approach are somewhat erratic since they depend strongly on the current mesh design. In contrast, the stresses given in Table 1 show convergence rates of algebraic type on a doubly logarithmic scale, and the results are highly accurate compared with the global energy norm approach and the energynorm-based goal-oriented approach.

In addition, we calculate the error bounds for the stresses obtained with the Green's function decomposition approach on uniform meshes based on (102) (see Fig. 18(b)). Here, we use the explicit error estimate de- fined in (28) for the energy norm errors used in (102) with the constants in (168). As seen, these error bounds produce an envelope which contains the exact solution, and which is quite narrow although the estimates for the local errors are based on explicit error estimators.

Next, we employ the energy-norm-based goal-oriented approach and the energy-norm-based Green's function decomposition approach, starting with a coarse mesh of $5 \times 5$ elements. We steer in each case the refinement process using (81) and the local error indicators given in (78) and (79), for the primal, the dual and the regular part problems. The refinement is performed using $\xi_{K}$ as above.

In Fig. 19 the final meshes obtained are displayed. As expected, the refinement of the energy-norm-based goal-oriented procedure concentrates on the region near the point of interest. The energy-norm-based Green's function decomposition approach can neglect this region because the fundamental solution is already the quasi-optimal choice for the region. Instead, the mesh is refined almost uniformly, which in this example is sufficient to approximate the regular part accurately.

While the Green's function decomposition approach is clearly very effective in this illustrative example, as already pointed out above, the method is however rather restrictive in that only analysis cases can be considered for which the fundamental solution is available. 


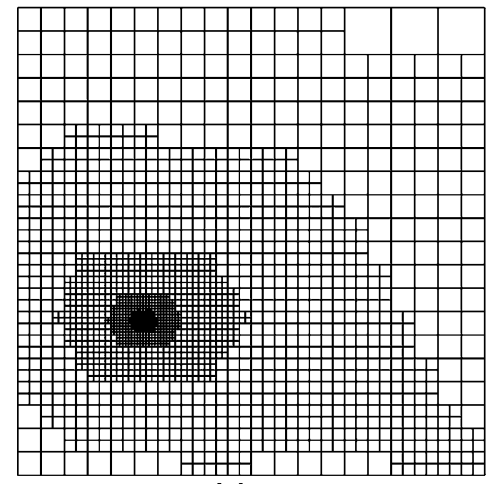

(a)

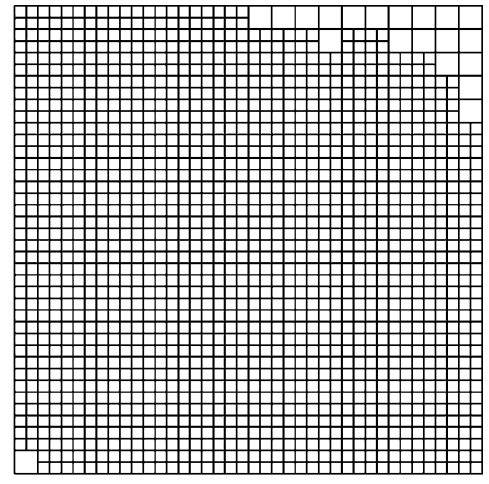

(b)

Fig. 19. Final meshes to approximate the stress $\sigma_{x x}$ at point A: (a) goal-oriented refinement, (b) refinement using the Green's function decomposition approach.

\section{Conclusions}

In this paper we reviewed some basic a posteriori error estimation techniques which broadly can be classified into global error estimators for the energy norm and goal-oriented error estimators to provide error estimates and error bounds for linear quantities of interest. We also discussed the case when the goal quantity is a point value, which normally poses a difficulty since the dual solution is not in the solution space.

The crucial issues of any error estimator relate to questions of reliability, accuracy and computational cost where, clearly, the definition what is an admissible cost always depends on the purpose of the computation [1]. As pointed out, there may be different reasons for using an error estimator. To only obtain an indication of the error or to drive a mesh adaptivity scheme with respect to the goal of the computation, explicit error indicators might be sufficient and these are generally quite inexpensive to use. On the other hand, to actually bound the error almost guaranteed in a suitable norm is at present only possible for certain problems, and then very expensive in practical analysis. However, it is also clear that the more accurate estimators have to be more expensive because a nearly exact estimate is close to finding the exact solution. The question is then whether it is not more effective in practice to simply use a very fine mesh.

A major point is that, in essence, error bounds are either guaranteed but, in practice for complex problems, hardly computable or they are computable but not guaranteed. Another major point is that while, in engineering practice, the analysis of shell structures constitutes a large percentage of all linear analyses, there are only some contributions that address bounding the error in suitable norms $[14,39,50,61,76-80]$ and goal-oriented procedures need still be further explored, in particular using mixed finite element discretizations [49].
Since sharp and effective error estimators are not yet available for many practical analyses, we are left with the common advice:

- The mesh should be reasonably fine and, ideally, solutions would be obtained for a coarse mesh and a finer mesh, for comparison purposes. Also, simple visual criteria to approximately assess the error might be used such as the stress iso-bands in ADINA.

- At all high stress gradients the mesh should be sufficiently fine. The results of the analysis are frequently most accurate when the relative error is uniform over the complete analysis domain.

- Integrated (averaged) quantities are usually more accurate than point values.

- In order to obtain highly accurate local quantities of interest, the mesh should of course be reasonably fine around the region of interest (and also in general in the areas of high stress gradients as mentioned already).

Future research work should address the development of actually implementable and practically useful improved error estimators that are applicable for a large class of problems. From an engineering point of view, inexpensive to calculate and guaranteed bounds on the error at every point of the structure, in the sense of our ideal-error-estimator-solution (see Fig. 1), would be very valuable. However, such error bound solution will likely be very difficult to achieve without a significant computational expense. In engineering practice, the calculation of the error measure should not be more expensive than the added expense to simply run a very fine mesh.

A quite promising approach is to use goal-oriented error measures in order to establish a coarse but still appropriate mesh in computationally intensive finite ele- 
ment solutions, notably in multi-physics and multi-scale analyses involving optimization. Here the premise is that an integrated quantity might be calculated with sufficient accuracy using a well-chosen coarse mesh in part of the domain. For example, considering a fluid flow structural interaction analysis, a coarse mesh representing the fluid might be sufficient to calculate the total force and moment on the structure [81]. And the computational expense to establish and use the coarse mesh of the fluid might be much less than the expense to use a very fine fluid mesh, in particular, if a structural optimization is required.

Hence, while the theory of error estimation has provided much valuable insight into the finite element solution process, many of the proposed techniques are at present only valuable to a limited extent in engineering practice.

Of course, throughout the paper we assumed that an appropriate mathematical model has been chosen and we only focused on the discretization errors arising in the finite element solution of this model (see Section 1). In practical engineering analysis, the errors arising due to an inappropriate mathematical model can naturally be much more significant than the error we have discussed in this paper $[1,82]$.

\section{Acknowledgement}

We would like to thank Donald Estep, Colorado State University, Ivo Babuška, University of Texas at Austin, Zhimin Zhang, Wayne State University, Jaime Peraire, MIT, Anthony Patera, MIT, and Slimane Adjerid, Virginia Tech, for their valuable comments on this paper.

The work of Thomas Grätsch was supported by the German Research Foundation (DFG) under contract GR 1894/2-1. We are grateful for this support.

\section{References}

[1] Bathe KJ. Finite element procedures. Englewood Cliffs, NJ: Prentice Hall; 1996.

[2] Szabó B, Babuška I. Finite element analysis. New York, USA: John Wiley \& Sons Inc; 1991.

[3] Verfürth R. A review of a posteriori error estimation and adaptive mesh refinement techniques. Chichester: John Wiley \& Sons; 1996.

[4] Estep D, Larson M, Williams R. Estimating the error of numerical solutions of systems of reaction-diffusion equations. Americal Mathematical Society, No. 696; 2000.

[5] Ainsworth M, Oden JT. A posteriori error estimation in finite element analysis. New York: John Wiley \& Sons; 2000.

[6] Babuška I, Strouboulis T. The finite element method and its reliability. Oxford: Oxford Science Publications; 2001.
[7] Brenner SC, Scott LR. The mathematical theory of finite element methods. Texts in applied mathematics Bd. 15. New York: Springer-Verlag; 1994.

[8] Johnson C. Numerical solution of partial differential equations by the finite element method. Cambridge: Cambridge University Press; 1987.

[9] Babuška I, Rheinboldt WC. A posteriori error estimates for the finite element method. Int J Numer Methods Engng 1978;12:1597-615.

[10] Babuška I, Miller AD. A feedback finite element method with a posteriori error estimation. Part I: The finite element method and some basic properties of the a posteriori error estimator. Comput Methods Appl Mech Engng 1987;61: $1-40$.

[11] Babuška I, Rheinboldt WC. Analysis of optimal finite element meshes in $R^{1}$. Math Comput 1978;33:435-63.

[12] Kelly DW, Gago OC, Zienkiewicz OC, Babuška I. A posteriori error analysis and adaptive processes in the finite element method: Part I-error analysis. Int J Numer Methods Engng 1983;19:1593-619.

[13] Johnson C, Hansbo P. Adaptive finite element methods in Computational Mechanics. Comput Methods Appl Mech Engng 1992;101:143-81.

[14] Stein E, Seifert B, Ohnimus S, Carstensen C. Adaptive finite element analysis of geometrically non-linear plates and shells, especially buckling. Int $\mathbf{J}$ Numer Methods Engng 1994;37:2631-55.

[15] Bank RE, Weiser A. Some a posteriori error estimators for elliptic partial differential equations. Math Comput 1985;44:283-301.

[16] Babuška I, Strouboulis T, Gangaraj SK. Guaranteed computable bounds for the exact error in the finite element solution. Part I: One-dimensional model problem. Comput Methods Appl Mech Engng 1999;176:51-79.

[17] Babuška I, Rheinboldt WC. Error estimates for adaptive finite element computations. SIAM J Numer Anal 1978;15: 736-54.

[18] Carstensen C, Funken S. Fully reliable localized error control in the FEM. SIAM J Sci Comput 2000;21:1465-84.

[19] Machiels L, Maday Y, Patera A. A flux-free nodal Neumann subproblem approach to output bounds for partial differential equations. CR Acad. Sci. Paris, vol. 330. Serie I; 2000. p. 249-54.

[20] Morin P, Nochetto RH, Siebert KG. Local problems on stars: a posteriori error estimators, convergence, and performance. Math Comput 2003;72:1067-97.

[21] Prudhomme S, Nobile F, Oden JT. Analysis of a subdomain-based error estimator for finite element approximations of elliptic problems. TICAM Report 02-34. The University of Texas at Austin, 2002. Numer Meth Part D and $\mathrm{E}$, in press.

[22] Sussman T, Bathe KJ. Studies of finite element procedures - stress band plots and the evaluation of finite element meshes. Engng Comput 1986;3:178-91.

[23] Zienkiewicz OC, Zhu JZ. A simple error estimator and adaptive procedure for practical engineering analysis. Int $\mathbf{J}$ Numer Methods Engng 1987;24:337-57.

[24] Carstensen C, Funken S. Averaging technique for FE-a posteriori error control in elasticity. Part I: conforming FEM. Comput Methods Appl Mech Engng 2001;190: 2483-98. 
[25] Zienkiewicz OC, Zhu JZ. The superconvergent patch recovery and a posteriori error estimates. Part 1: the recovery technique. Int J Numer Methods Engng 1992; 33:1331-64.

[26] Zienkiewicz OC, Zhu JZ. The superconvergent patch recovery and a posteriori error estimates. Part 2: Error estimates and adaptivity. Int $\mathbf{J}$ Numer Methods Engng 1992;33:1365-82.

[27] Zhang Z, Naga A. A new finite element gradient recovery method: superconvergence property. SIAM J Sci Comput, in press.

[28] Xu J, Zhang Z. Analysis of recovery type a posteriori error estimators for mildly structured grids. Math Comput 2004;73:1139-52.

[29] Hiller JF, Bathe KJ. On higher-order-accuracy points in isoparametric finite element analysis and an application to error assessment. Comput Struct 2001;79:1275-85.

[30] Babuška I, Miller AD. The post-processing approach in the finite element method, I: calculations of displacements, stresses and other higher derivatives of the displacement. Int J Numer Methods Engng 1984;20:1085-109.

[31] Eriksson K, Johnson C. Adaptive finite element methods for parabolic problems I: a linear model problem. SIAM J Numer Anal 1991;28:43-77.

[32] Estep D. A posteriori error bounds and global error control for approximations of ordinary differential equations. SIAM J Numer Anal 1995;32:1-48.

[33] Eriksson K, Estep D, Hansbo P, Johnson C. Introduction to adaptive methods for differential equations. Acta Numer 1995:105-58.

[34] Becker R, Rannacher R. A feed-back approach to error control in finite element methods: basic analysis and examples. East-West J Numer Math 1996;4: 237-64.

[35] Rannacher R, Suttmeier FT. A feed-back approach to error control in finite element methods: application to linear elasticity. Comput Mech 1997;19:434-46.

[36] Giles MB, Larson MG, Levenstam JM, Süli E. Adaptive error control for finite element approximations of the lift and drag coefficients in viscous flow. Report NA-97/06. Oxford University Computing Laboratory; 1997.

[37] Paraschivoiu M, Peraire J, Patera A. A posteriori finite element bounds for linear-functional outputs of elliptic partial differential equations. Comput Methods Appl Mech Engng 1997;150:289-312.

[38] Paraschivoiu M, Patera A. A hierarchical duality approach to bounds for the outputs of partial differential equations. Comput Methods Appl Mech Engng 1998;158:389-407.

[39] Çirak F, Ramm E. A posteriori error estimation and adaptivity for linear elasticity using the reciprocal theorem. Comput Methods Appl Mech Engng 1998; 156:351-62.

[40] Pierce NA, Giles MB. Adjoint recovery of superconvergent functionals from PDE approximations. Siam Rev 2000;42: 247-64.

[41] Giles MB, Suli E. Adjoint methods for PDEs: a posteriori error analysis and postprocessing by duality. Acta Numer 2002:145-236.

[42] Tottenham H. Basic principles. In: Tottenham H, Brebbia C, editors. Finite element techniques in structural mechanics. Southampton: Southampton University Press; 1970.
[43] Adjerid S, Salim M. Even-odd goal-oriented a posteriori error estimation for elliptic problems. Appl Numer Math, in press.

[44] Prudhomme S, Oden JT. On goal-oriented error estimation for elliptic problems: application to the control of pointwise errors. Comput Methods Appl Mech Engng 1999;176:313-31.

[45] Oden JT, Prudhomme S. Goal-oriented error estimation and adaptivity for the finite element method. Comput Math Appl 2001;41:735-56.

[46] Babuška I, Miller AD. The post-processing approach in the finite element method, III: a posteriori error estimates and adaptive mesh selection. Int $\mathbf{J}$ Numer Methods Engng 1984;20:2311-24.

[47] Ohnimus S, Stein E, Walhorn E. Local error estimates of FEM for displacements and stresses in linear elasticity by solving local Neumann problems. Int J Numer Methods Engng 2001;52:727-46.

[48] Bangerth W, Rannacher R. Adaptive finite element methods for differential equations. Basel: Birkhäuser; 2003.

[49] Grätsch T, Bathe KJ. Influence functions and goaloriented error estimation for finite element analysis of shell structures. Int J Numer Methods Engng, submitted for publication.

[50] Chapelle D, Bathe KJ. The finite element analysis of shells-fundamentals. Berlin: Springer-Verlag; 2003.

[51] Bathe KJ, Lee PS, Hiller JF. Towards improving the MITC9 shell element. Comput Struct 2003;81: 1085-97.

[52] Bathe KJ, Iosilevich A, Chapelle D. An evaluation of the MITC shell elements. Comput Struct 2000;75:1-30.

[53] Wang S, Sloan IH, Kelly DW. Computable error bounds for pointwise derivatives of a Neumann problem. IMA J Numer Anal 1998;18:251-71.

[54] Cao D, Kelly DW, Sloan IH. Local error bounds for postprocessed finite element calculations. Int J Numer Methods Engng 1999;45:1085-98.

[55] Grätsch T, Hartmann F. Finite element recovery techniques for local quantities of linear problems using fundamental solutions. Comput Mech 2003;33:15-21.

[56] Hartmann F, Katz C. Structural mechanics with finite elements. Berlin: Springer-Verlag; 2004.

[57] Sauer-Budge AM, Bonet J, Huerta A, Peraire J. Computing bounds for linear functionals of exact solutions to Poisson's equation. SIAM J Numer Anal, in press.

[58] Sauer-Budge AM, Peraire J. Computing bounds for linear functionals of exact solutions to the advection-diffusionreaction equation. SIAM J Sci Comput, in press.

[59] Prud'homme C, Rovas DV, Veroy K, Machiels L, Maday Y, Patera A, Torinici G. Reliable real-time solution of parametrized partial differential equations: reducedbasis output bound methods. ASME 2002;124:7080.

[60] Estep D, Holst M, Larson M. Generalized Green's functions and the effective domain of influence. SIAM J Sci Comput, in press.

[61] Prudhomme S, Oden JT, Westermann T, Bass J, Botkin M. Practical methods for a posteriori error estimation in engineering applications. Int $\mathbf{J}$ Numer Methods Engng 2003;56:1193-224. 
[62] Caloz G, Rappaz J. Numerical analysis for nonlinear and bifurcation problems, Part 2. Ciarlet PG, Lions JL, editors. Handbook of numerical analysis, Vol. V. Amsterdam: Elsevier; 1997. p. 487-638.

[63] Larsson F, Hansbo P, Runesson K. Strategies for computing goal-oriented a posteriori error measures in nonlinear elasticity. Int J Numer Methods Engng 2002;55: 879-94.

[64] Çirak F, Ramm E. A posteriori error estimation and adaptivity for elastoplasticity using the reciprocal theorem. Int J Numer Methods Engng 2000;47:379-93.

[65] Larsson F, Runesson K, Hansbo P. Time finite elements and error computation for (visco) plasticity with hardening or softening. Int J Numer Methods Engng 2003;56: 2213-32.

[66] Lee NS, Bathe KJ. Error indicators and adaptive remeshing in large deformation finite element analysis. Finite Elem Anal Des 1994;16:99-139.

[67] Gallimard L, Ladèveze P, Pelle JP. Error estimation and adaptivity in elastoplasticity. Int $\mathbf{J}$ Numer Methods Engng 1996;39:189-217.

[68] Rannacher R, Suttmeier FT. A posteriori error estimation and mesh adaption for finite element models in elastoplasticity. Comput Methods Appl Mech Engng 1999;176: 333-61.

[69] Süli E, Houston P. Finite element methods for hyperbolic problems: a posteriori error analyses and adaptivity. Invited Lecture at State of the Art in Numerical Analysis Conference. York, 1-4 April, 1996. Oxford University Computing Laboratory, Report 96/06, 1996.

[70] Schleupen A, Ramm E. Local and global error estimation in linear structural dynamics. Comp Struct 2000;76:741756.

[71] Wiberg NE, Li XD. A postprocessed error estimate and an adaptive procedure for the semidiscrete finite element method in dynamic analysis. Int J Numer Methods Engng 1994;37:3585-603.

[72] Brezzi F, Fortin M. Mixed and hybrid finite elements methods. New York: Springer-Verlag; 1991.

[73] Bathe KJ. The inf-sup condition and its evaluation for mixed finite element methods. Comput Struct 2001;79: 243-52. p. 971.

[74] Brezzi F, Bathe KJ. A discourse on the stability conditions for mixed finite element formulations. Comput Methods Appl Mech Engng 1990;82:27-57.

[75] Braess D, Klaas O, Niekamp R, Stein E, Wobschal F. Error indicators for mixed finite elements in 2-dimensional linear elasticity. Comput Methods Appl Mech Engng 1995; 127:345-56.

[76] Hiller JF, Bathe KJ. Measuring convergence of mixed finite element discretizations: An application to shell structures. Comput Struct 2003;81:639-54.

[77] Huerta A, Rodriguez-Ferran A, Diez P, Sarrate J. Adaptive finite element strategies based on error assessment. Int J Numer Methods Engng 1999;46:1803-18.

[78] Yazdani AA, Riggs HR, Tessler A. Stress recovery and error estimation for shell structures. Int J Numer Methods Engng 2000;47:1825-40.

[79] Düster A, Bröker H, Rank E. The p-version of the finite element method for three-dimensional curved thin walled structures. Int J Numer Methods Engng 2001;52: 673-703.

[80] Oden JT, Prudhomme S, Westermann T, Bass J, Botkin M. Error estimation of eigenfrequencies for elasticity and shell problems. Math Mod Methods Appl S 2003;13: 323-44.

[81] Grätsch T, Bathe KJ. Goal-oriented error estimation in the analysis of fluid flows with structural interactions, in preparation.

[82] Bucalem M, Bathe KJ. Hierarchical modeling in finite element analysis. Springer-Verlag, in preparation. 